INSTITUTO DE MATEMÁTICA E ESTATISTTICA - U.S.P.

\title{
Distribuição de Tolerância da Mistura de Dois Estimulos Independentes
}

DISSERTAÇĀO DE MESTRADO

GABRIELA STANGENHAUS

Orientador: Dr. EUCLIDES C. DE LIMA FILHO 


\section{AGRADECIMENTOS}

Ao Prof. Dr. Euclides Custódio de Iima Filho, os ensinamentos que nos miniatrou e o apolo dispensado durante o eatudo desta dissertação.

Ao Prof. Dr. Odelar Ie1te Linhares, o incentivo conatante e o esforco para not proporcionar excelen tea condiçõe de trabaliho.

Aos colegar do Departamento a atenção a cola boraço sempre diapensadas.

A CAPES, o auxillo concedido para a publicação deate trabalho.

Ao Centro de Processamento de Dados do Campus de São Carlos, da USP, o proceasamento dos programas apresentados neste trabalbo.

Ao José, mer amigo companhe1ro, o estimulo e sugeatões sempre oportunas. 


\section{SUMMARY}

TOLERANCE DISTRIBUTION FOR THE

\section{MIXTURE OF TWO INDEPENDENT STIMULI}

This disartation deala with the analyais of a mixture of two independent stimuli based on quantal data.

A method is presented to eatimate the parameters of the tolerance distribution, under the hypotheris that it is a Bivariate Normal. The method is easentially due to Hewlett and Plackett [10]. It estimates the two means and variances using the (Normal) marginal tolerance distributions and the method of probite, which 1s also preaented here.

The maximum-1ikelihood estimate of the correlation coefficient is obtained through the Newton iterative method.

A aet of computer programs is also presented, to enable the use of the method by non-apecialiats. 


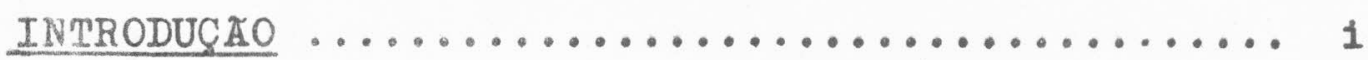

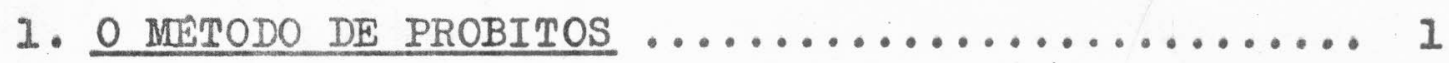

1.1. Considerações iniciaí ................. I

1.2. Distribuição de tolerância .............. 1

1.3. Transformação através de probitos ......... 2

1.4. Eatimação dos parâmetros de uma diatribuição de tolerância ...................... 4

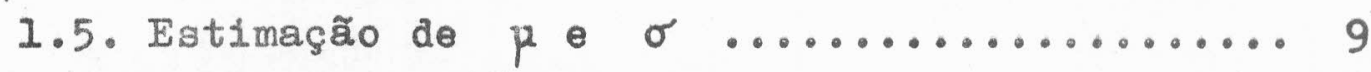

1.6. Teste de aderência ...................... 13

1.7. Intervalos de conflança .............. 15

1.8. Comparação entre duas diatribuiçõea de tole-

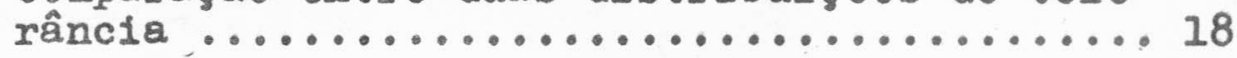

2. DISTRIBUICAO DE TOLERANCIA DA MISTURA DE DOIS ES-

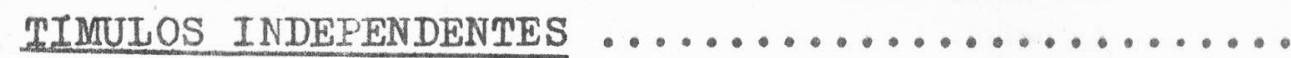

2.1. Determinação da diatríbuição de tolerância de uma mistura a partir da distribuição de tole rância de seus componentes .............. 22

2.2. Uso da distribuição Normal como distribuição da tolerância da mistura de dois estímulos

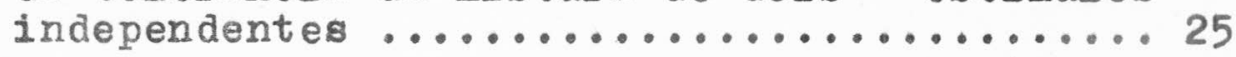

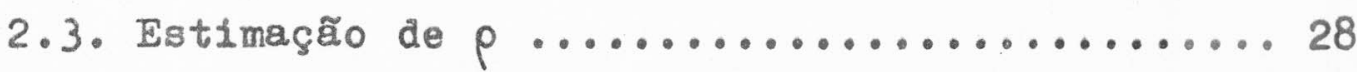

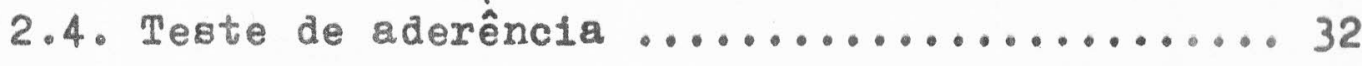

2.5. Roteiro para a análise da mistura de do1s es

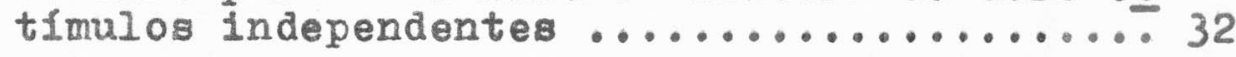

2.6. Alternativas para a estimação de $\mu_{1}, \mu_{2}, \sigma_{1}$

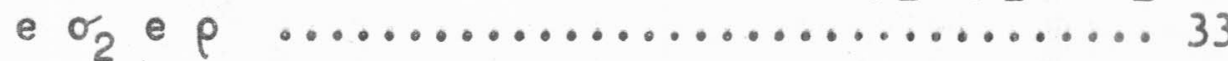

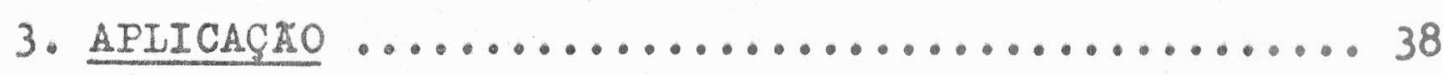

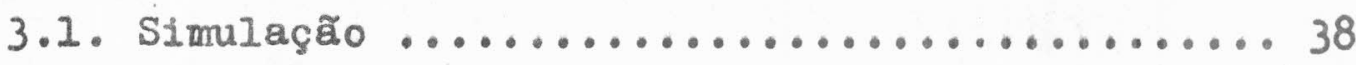

3.2. Estimação de $\mu_{1}, \mu_{2}, \sigma_{1}, \sigma_{2}$ e $\rho \ldots . . . . .38$ 3.3. Teste de Aderência .................... 40 3.4. Convergênc1a do processo 1terativo de Newton 41

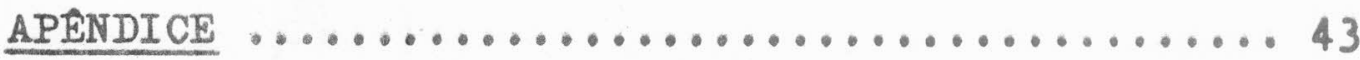

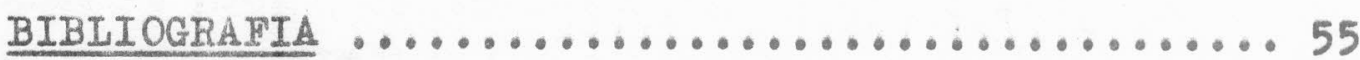




\section{INTRODUCEO}

Ocorrem na prática experimentos cujos resultados ão de caráter qualitatiro (não quantificáveí). Por exemplo, se desejamos estudar a eficácla de um inseticida, tomamos várlos lotes de insetos aos quais aplicamo doses dis tintas do inseticida. Cada inseto pode, segundo nose ob Bervaça, sex considerado como morto ou sobrevivente. Co mo outro exemplo, consideremos cápsulas de fulminato de mercúrio (detonadores) que são submet1das a choque mecânico de intensidade conhecida. Cada prova reaultará ou em explosão ou em não-explosão.

Os dols experimentos, um de Biologia, o outro de Enge nharia, têm em comum o típo de resultado, que dicotômí co (resposta, não-resposta). Também são passírels de se rem igualmente formulados: a lotes de unidades experimen tais (Insetos, cápsulas de fulminato de mercúrio) aฮ̂́ apli cados estímulos (inseticida, choque mecân1co) em vários niveis (doses, intensidades), obtendo-se resposta (morte, explosão) ou não-resposta (sobrevivêncla, falha). Tal tipo de resposta é dita dicotômica ou quantal.

A análise de experimentos com respostas quantais é um problema resolvido, quando consideramo apenas um estimu 10.

Todavia, pode ocorrer a necossidade de estudar a ação simultânea de do1s estímulos. A ação simultânea de dols inseticidas, por exemplo, bem pode ser de interesse prático.

Dojs estimulos aplicados simultaneamente constituem uma mistura de estimulos a são ditos independentes ee eles agem sobre siatemas distintos a ação de um estímulo nåo interfere na ação do outro.

Um experimento com uma mistura é felto aplicando-se a diversos lotes de unidades experimentais niveis da mistu ra e observando-se, em cada lote, o número de resposta.s. Suponhamos que um certo lote contém $n_{1}$ unidader exper1 menta18, das quais $r_{1} \leqslant n_{1}$ responderam a mistura. A and I1se estatística dos resultados experimental refere-se 
à relação entre a pxoporçõe observadar $p_{1}=r_{1} / n_{1}$ e or nivela da mistura aplicados aos diversos loter. Para Interpretar dados desse t1po em termos quantitativos, tor na-вe necessário adotarmos um modelo matemático para relacionar os nive16 da miatura 0 as probabilidadea de res posta (distribuição de tolerânc1a). Essa distribulção de tolerância iré envolver um conjunto de parâmetros a ae rem estimados a partir doa dador experimentais.

Neste trabalho trataremos da determinação da distri buiçăo de tolerâncla da mistura de dole estímulos independenter A e B. Suporemos quo a distribuiço de to lerêncla da miatura é Normal bl dimensional.

No Capitulo 1 apresentamoa o método de probitos, que se preata a estimar a média e a variância de uma distribuição Normal, quando os dados são quanta1s.

- Capitulo 2 consiste na determinaça da diotribulgão de tolerância da mistura de dols estimulns independentes. Primeiramente determinamos a distribuição de tolerância da mistura a partíx da distribujção de tolerância de seu componentes. Em aeguida tratamos da estimaça dos parâmetror da distribuiçåo de tolexância no caro em que eata. 6 suposta Normal bidimensional. As médias o as variânc1a dos est1mulos aฐ̃o estimadas através do método depro bitor o o coeflclente de correlağão é estimado, basica mente, pelo método apresentado por Hewlett e Plackett [8]. Para finalizar apresentamo algun métodor adicionais pa ra est1mar os parâmetros da distxibuı̣ço normal bidimenaional, no caso de respoatas quantais.

No capitulo 3 fazemos aplicações da teoria apresentada nos capitulos 1 - 2 a dados simulados em computa dor.

Apresentamor em um apêndice dolı programas para compu tador, que elaboramos para a estimação dos parâmetron em uma anál1вe de misturaø. O primelro programa calcula os valores eatímados da média e da variância pelo método de probitos. 0 segundo calcula a estimativa do coeficiente de correlaçåo. A Incluą̃o doa programa fol felta para permitir a peøsoa nåo 1njc1ada em programaça o emprogo de computadore na colugăo do problemas rea1. 


\section{O METODO DE PROBITOS}

\subsection{Cona1deracõos Inicia1日}

Apresentaremo algumas definições que serão frequen temente út1lizadar ao longo deste trabalho.

Em certas experiências existem đuas componentea a sexem conalderadar:

a. EstImulo - uma ação que produz determinada reaposta.

b. Unidade experimental - elemento ao qual é aplicado o estimulo.

o estímulo é eplicado à unidade experimental numa in tensidade especificada e como reaultado obtém-ae, ou não, uma resposta. A capacidade que o estimulo possul de pro duzix resposta.s denominada potência do estímulo.

Quando realizamos experiências cujo resultado é a respoata a um estimulo aplicado a uma unidade experimental, Irequentemente é imposaivel ou impraticável obter-ge uma medida quantitativa dessa resposta. 0 que se pode saber apbs a aplicaç̃o do estlmulo é se produziu ou não um efeito especificedo. Por exemplo, ao injetar-ae certa dose de veneno em um lote de cobalas, verifica-se, após dez minutos, quantas morreram. Esse t1po do respos ta denomina-re dicotômica ou quantal.

Quando a resposta é quantal, sua ocorrência 1rá dępender de Intensidade do estimulo aplicado (nivel do eatImulo). Associado a cada unidade experimental existe um nIvel abaixo do qual ela não reaponde ao eatímulo e aclma do qual ela reaponde. Eare nivel denomina-se "tole rância" da unjdade experimental.

\subsection{Distribuicão de tolerânc1a}

A tolerância varia do unidade experimental para und dade experimental. Portanto, para estudarmos a potêncie de um estrmulo é necessário conhecermo a distrubulģão de tolexêncla.

A medida do estimulo aerá expressa por $\lambda$ altr 1 bulção de tolerância, por: 


$$
d P=\mathscr{P}(\lambda) d \lambda
$$

Então, se um nIteI $\lambda_{1}$ do otImulo aplicado a uma un1dade experimental, a probab111dade de resposta a esse nIVel do etsimulo

$$
P_{1}=\int_{0}^{\lambda_{1}} f(\lambda) d \lambda
$$

Suporemos que o eat1mulo fo maldo por uma quant1dado que raria de $0 a+\infty$. Assin,

$$
\int_{0}^{+\infty} P(\lambda) d \lambda=1
$$

A experiência tem mostrado que $f(\lambda)$ ć geralmente as 1 métrice, quando medida ne escals natural e que através de uma tranøformação adequada de $\lambda$ obter-ae uma diatr1bu1 gão aImétrica, que é aproximedamente Norma1. Denotare mos por $x$ - valor transformado de $\lambda$ orá denomínado de nivel transformado.

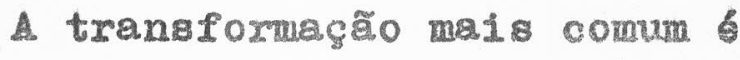

$$
x=\log _{10} x
$$

Dcoxxe também com fraguêncta a transformagão

$$
x=\lambda^{1} \quad 1 \leq 1
$$

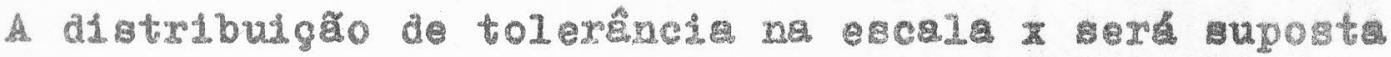
Normal, 1 to 6, sua funço de denaidado á dada por

$$
\begin{aligned}
& \text { (1.2.3) } f(x)=\frac{1}{\sigma \sqrt{2 \pi}} \quad \begin{aligned}
2 \sigma & -\infty<x<+\infty \\
& -\infty<p<+\infty \\
&
\end{aligned} \\
& \begin{aligned}
\frac{(x-\mu)^{2}}{2 \sigma^{2}} & -\infty<x<+\infty \\
-\infty<p<+\infty & <-\infty>0
\end{aligned}
\end{aligned}
$$

A deno1dade $(1.2 .3)$ geré denotade por $N(\mu ; \sigma)$

\subsection{Tranaformacão através de probitos}

Pare estudar-se a distríbulgåo de tolerêncla de um estlmulo, escolhem-so diversos nIVal dosse erimulo o apllca-re cade nírel a un lote do undades experluental. 
Observa-se então, em cada lote, a proporça de unidadea experimentais que respondeu ao nivel aplicado. Normalmen te a relação entre nivel e reaposta é expresą por uma curva algmbide asalmétrica, como já fol dito.

Quando se aplica um eatimulo a um lote de unidades ex perimenta1ョ, 1rão responder ao estímulo aquelas unldadea cuja tolerância é Inferior ao nivel aplicado. Eare fato uma Inerente variabilidade na suacetibllidade entre as unidades experimenta1 são responá́veí pela forma siomólde da curva.

Existe um inconveniente em ae eatudar diretamente esaa curva sigmólde. Ela é assint6t1ca na região em que a percentagem de resposta se aproxima de $100 \%$, tornando d 1 ficll a determinação do menor nivel que age sobre cerca de $100 \%$ da população estudada. Alem do ma1s, a estimação dos parâmetros da distribuição de tolerânc1a é traba 1hosa. Para superar esas dificuldades, BIIss [2] propôs a traneformação dos níveis em logar1tmos dectmais dos nívels e as percentagens de resposta nas abscis sas correapondentes da d1stribu1ço Normal com méda 5 e variânc1a 1. Esså unldades foram denominadas de probltos. O probito é uma modificação do N.E.D. (normal equiva lent deviate), definido por Gaddum, que é o nivel transformado esperado numa distribulção normal com média 0 var1ânc1a 1, corregpondente a uma proporgão. Bl1sa achou conveniente adotar a média 5 para evitar valorem negativos.

Obtemos com essa tranıformação pontos que podem ser repreaentados por uma reta. Assim, a ankilse da potêncla dos estimulos pode ser realizada atravéa dos coeficlenter angular e linear da reta.

Seja $P$ a probabilidade de resposta a un certo nivel transformado $x$; então o problto de $P$ é dado por $Y$, onde

$$
P=\frac{1}{\sqrt{2 \pi}} \int_{-\infty}^{Y-5} \cdot \frac{v^{2}}{2} d v \quad(1.3 .1)
$$

Se (1.2.3) representa a distribu1ção de tolerẩncia, então a probabilidade eaperada de resposta um alvel 
trangformado $\times$ é,

$$
P=\frac{1}{\sigma \sqrt{2 \pi}} \int_{-\infty}^{x} e^{-\frac{(v-\mu)^{2}}{2 \sigma^{2}}} d v
$$

Comparando $(1.3 .1)$ e.(1.3.2) com respeito a weama pro porção $P$, obtemos:

$$
Y=5+\frac{1}{\sigma}(x-\mu)
$$

De (1.3.3) concluimos que a relação entre o probito da proporção esperada de resposta o nivel tranøformado é Iinear. Assim, através de probitos, dados experimentals podem aer utilizados para fornecer uma estimativa de $\mu$ e $\sigma$.

1.4. Estimação dos parâmetros de uma distribuicão de toIerância

Suponhamos que a diatribuição de tolerância de um estímulo seja dada por (1.2.1), que depende do parâmetro $\theta$, e que a probabilidade de uma unidade experimental res ponder ao nivel $\lambda_{1}$ do estimulo seja dada por $(1.2 .2)$.*

Se um estímulo é aplicado ao nivel $\lambda_{1}$ a um lote com $n_{1}$ unidades experimentals $\theta$ se as respostas das unidades ex perimentais aฮ̃o 1ndependentes, então a probabilidade de que $r_{1}$ unidades experimentais respondam ao estimulo é da da pela distribuição binomial,

$$
\begin{aligned}
-P\left(r_{1}\right)= & \left(\begin{array}{l}
n_{1} \\
r_{1}
\end{array}\right) P_{1}^{r_{1}} Q_{1}^{n_{1}-r_{1}} \quad r_{1}=0,1, \ldots, n_{1} \\
Q_{1} & =1-P_{1}
\end{aligned}
$$

Suponha-se agora que uma sérle de k nivelo de um estí mulo săo teṣtados em uma certa experiência. A partir dos resultados experimenta1s, ut1lizando o método de máxima-verosвimilhança, verão estımados os parદ́metros de $f(\lambda)$.

A função de verossimilhança da amostra 6,

$$
I_{1}=\prod_{1=1}^{k}\left(\begin{array}{l}
n_{1} \\
r_{1}
\end{array}\right) P_{1}^{r_{1}} Q_{1}^{n_{1}-r_{1}}
$$


O estlmador de máxima verosimilhança de $\theta$ a a a vução da equação

$$
\frac{\partial I_{1}}{\partial \theta}=0
$$

Log $I_{1}$ e $I_{1}$ tem o mesmo ponto de máximo, pole Log $I_{1}$ 6 uma fungão crescente de $I_{1}$. Podemos então determinsr - estimador de $\theta$ através da equaço:

Mas,

$$
\frac{\partial \log I_{1}}{\partial \theta}=0
$$

$I=\log I_{1}=\sum_{1=1}^{k} \log \left(r_{1}^{n}\right)+\sum_{1=1}^{k} r_{1} \log P_{1}+\sum_{1=1}^{k}\left(n_{1}-r_{1}\right) \log Q_{1}$

Então,

$$
\begin{aligned}
\frac{\partial I}{\partial \theta} & =\sum_{1=1}^{k} \frac{r_{1}}{P_{1}} \frac{\partial P_{1}}{\partial \theta}+\sum_{1=1}^{k} \frac{\left(n_{1}-r_{1}\right)}{Q_{1}} \frac{\partial Q_{1}}{\partial \theta}= \\
& =\sum_{1=1}^{k} \frac{r_{1}}{P_{1}} \frac{\partial P_{1}}{\partial \theta}-\frac{\left(n_{1}-r_{1}\right)}{Q_{1}} \frac{\partial\left(1-P_{1}\right)}{\partial \theta}= \\
& =\sum_{1=1}^{k} \frac{\left(r_{1}-n_{1} P_{1}\right)}{P_{1} Q_{1}} \frac{\partial P_{1}}{\partial \theta}=\sum_{1=1}^{k} \frac{n_{1}\left(p_{1}-P_{1}\right)}{P_{1} Q_{1}} \frac{\partial P_{1}}{\partial \theta}
\end{aligned}
$$

Obtemos asa1m a equação:

$$
\sum_{1=1}^{k} \frac{n_{1}\left(p_{1}-P_{1}\right)}{P_{1} Q_{1}} \quad \frac{\partial P_{1}}{\partial \theta}=0
$$

em que $p_{1}=r_{1} / n_{1}$ é uma estimativa empirica de $P_{1}$.

Se a distribuigão de tolerância envolve mais de um pa râmetro, os estimadores de máxima verosaimilhança dos pa râmetros serão dados pela solução de um sistema de equa çõea, em que, para cada parâmetro, tem-ge uma equação do tipo $(1.4 .2)$

Normalmente é 1mposalvel resolver esses sistemas atra vés de métodos exatos. Entretanto podemos ut111zar méto dos iterativos que convergem para a solução. 
Suponhamos que a distribuiça de tolerância depende de dois parâmetros $\theta$ e $\varnothing$. Seus estimadores de máxima ve rosaimilhança são a solução do sistema:

$$
\left\{\begin{array}{l}
\sum_{1=1}^{k} \frac{-n_{1}\left(p_{1}-P_{1}\right)}{P_{1} Q_{1}} \frac{\partial P_{1}}{\partial \theta}=0 \\
\sum_{1=1}^{k} \frac{n_{1}\left(p_{1}-P_{1}\right)}{P_{1} Q_{1}} \frac{\partial P_{1}}{\partial \varnothing}=0
\end{array}\right.
$$

Através do método iterativo de Newton obtemos estimativas de $\theta$ e $\varnothing$ que convergem para as estimativas de máxima-verossimilhança. Suponhamos que $\theta_{1}$ e $\phi_{1}$ são as prí meiras aproximações das soluçõea do s1stema (1.4.3), ob tidas através de método gráfico (Finney, [5], pg.28) ou outro processo. Deaenvolvendo-se $\partial I / \partial \theta$, $\partial I / \partial \phi$ em séries de Taylor até as derivadas de $l$ ordem temos,

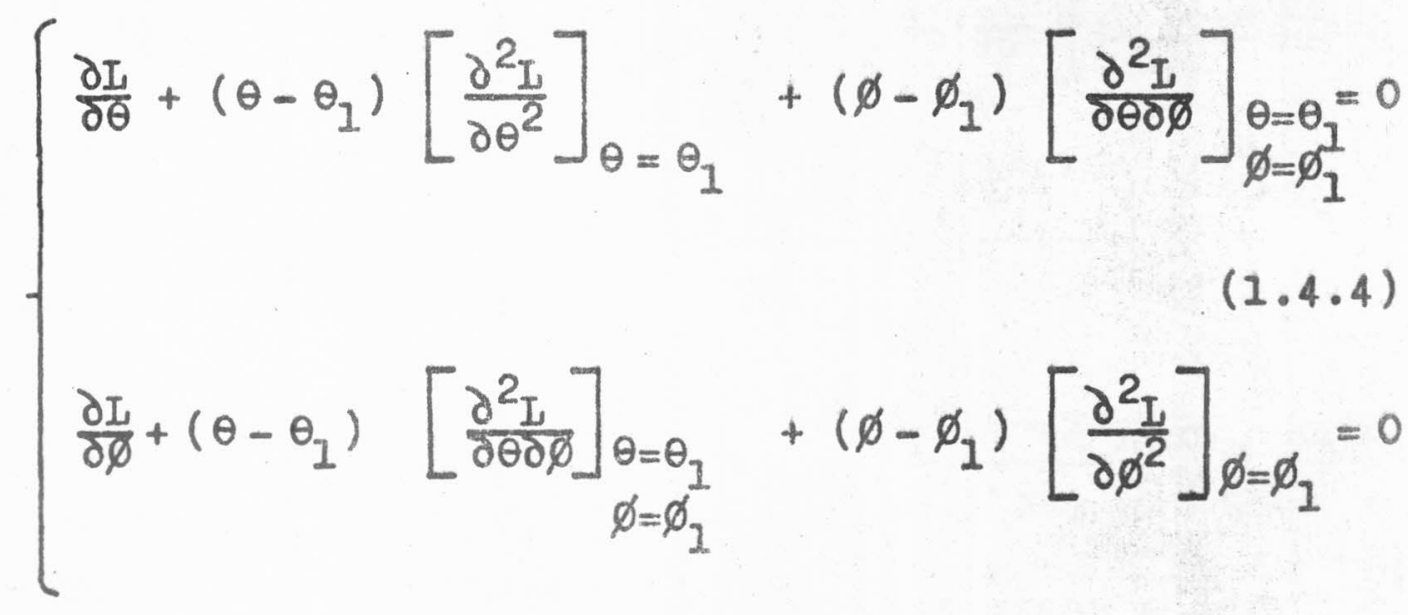

Considerando-se (1.4.1) obtemos as seguintes deriva das

$$
\begin{aligned}
\frac{\partial^{2} I}{\partial \theta^{2}}= & \sum_{1=1}^{k}\left[\frac{-n_{1}}{P_{1} Q_{1}}\left(\frac{\partial P_{1}}{\partial \theta}\right)^{2}-\left[\left(\frac{\partial P_{1}}{\partial \theta}\right)^{2}-2 P_{1}\left(\frac{\partial P_{1}}{\partial \theta}\right)^{2}\right] \times\left[\frac{n_{1}\left(P_{1}-P_{1}\right)}{P_{1}^{2} Q_{1}^{2}}\right]+\right. \\
& \left.+\frac{n_{1}\left(P_{1}-P_{1}\right)}{P_{1} Q_{1}} \frac{\partial^{2} P_{1}}{\partial \theta_{1}^{2}}\right]
\end{aligned}
$$




$$
\begin{aligned}
\frac{\partial^{2} I_{1}}{\partial \phi^{2}}= & \sum_{1=1}^{k}\left[\frac{-n_{1}}{P_{1} Q_{1}}\left(\frac{\partial P_{1}}{\partial \varnothing}\right)^{2}-\left[\left(\frac{\partial P_{1}}{\partial \varnothing}\right)^{2}-2 P_{1}\left(\frac{\partial P_{1}}{\partial \varnothing}\right)^{2}\right] \times\left[\frac{n_{1}\left(P_{1}-P_{1}\right)}{P_{1}^{2} Q_{1}^{2}}\right]+\right. \\
& \left.+\frac{n_{1}\left(p_{1}-P_{1}\right)}{P_{1} Q_{1}} \frac{\partial^{2} P_{1}}{\partial \phi^{2}}\right]
\end{aligned}
$$

0

$$
\begin{aligned}
\frac{\partial^{2} I}{\partial \theta \partial \varnothing}= & \sum_{1=1}^{k}\left[\frac{-n_{1}}{P_{1}^{Q_{1}}}\left(\frac{\partial P_{1}}{\partial \theta}\right)\left(\frac{\partial P_{1}}{\partial \phi}\right)\left[\frac{\left(p_{1}-P_{1}\right)\left(1-2 P_{1}\right)}{P_{1} Q_{1}}+1\right]+\right. \\
& \left.+\frac{n_{1}\left(p_{1}-P_{1}\right)}{P_{1}^{Q_{1}}} \frac{\partial^{2} P_{1}}{\partial \theta \partial \varnothing}\right]
\end{aligned}
$$

Subatituindo-se os valorea observados $p_{1}$ pelog valo w res espersdos $P_{i}$, temos

$$
\begin{aligned}
& \frac{\partial^{2} I}{\partial \theta^{2}}=\sum_{1=1}^{k} \frac{-n_{1}}{P_{1} Q_{1}}\left(\frac{\partial P_{1}}{\partial \theta}\right)^{2} \\
& \frac{\partial^{2} I}{\partial \phi^{2}}=\sum_{1=1}^{k} \frac{-n_{1}}{P_{1} Q_{1}}\left(\frac{\partial P_{1}}{\partial \phi}\right)^{2} \\
& \frac{\partial^{2} I}{\partial \theta \partial \varnothing}=\sum_{i=1}^{k} \frac{-n_{1}}{P_{1} Q_{1}}\left(\frac{\partial P_{1}}{\partial \theta}\right)\left(\frac{\partial P_{1}}{\partial \varnothing}\right)
\end{aligned}
$$

Ao fazer-ae tal substituição comete-se um erro que con verge em probabilidade para 0, pola o método de máxima ve rossimilhanģa garante a consiatência dos estimadores.

Substituindo $(1.4 .6),(1.4 .7) \cdot(1.4 .8)$ an $(1.4 .4)$ rem 


$$
\begin{aligned}
& \delta \theta_{1} \sum_{1=1}^{k} \frac{n_{1}}{P_{1} Q_{1}}\left[\left(\frac{\partial P_{1}}{\partial \theta}\right)^{2}\right]_{\theta=\theta_{1}}+\delta \phi_{1} \sum_{1=1}^{k} \frac{n_{1}}{P_{1} Q_{1}}\left[\frac{\partial P_{1}}{\partial \theta}\right]_{\theta=\theta_{1}} x \\
& x\left[\frac{\partial P_{1}}{\partial \phi}\right]_{\phi=\phi_{1}}=\sum_{1=1}^{k} \frac{n_{1}\left(p_{1}-P_{1}\right)}{P_{1} Q_{1}}\left[\frac{\partial P_{1}}{\partial \theta}\right]_{\theta=\theta_{1}} \\
& \delta \theta_{1} \sum_{1=1}^{K} \frac{n_{1}}{P_{1} Q_{1}}\left[\frac{\partial P_{1}}{\partial \theta}\right]_{\theta=\theta_{1}}\left[\frac{\partial P_{1}}{\partial \varnothing}\right]_{\phi=\phi_{1}}+\delta \phi_{1} \sum_{1=1}^{K} \frac{n_{1}}{P_{1} \theta_{1}}\left[\left(\frac{\partial P_{1}}{\partial \varnothing}\right)^{2}\right]_{\phi=\phi_{1}}= \\
& =\sum_{i=1}^{k} \frac{n_{1}\left(p_{1}-P_{1}\right)}{P_{1} Q_{1}}\left[\frac{\partial P_{1}}{\partial \phi}\right]_{\phi=\phi_{1}}
\end{aligned}
$$

em que $\delta \theta_{1}=\theta-\theta_{1}$ e $\delta \phi_{1}=\varnothing-\phi_{1}$

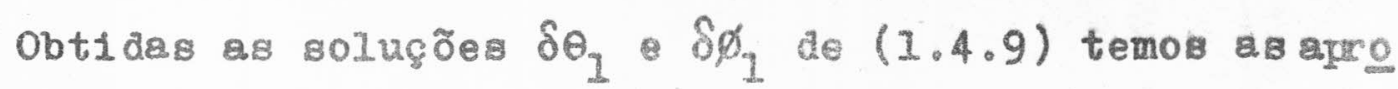
ximações melhoradas

$$
\theta_{2}=\theta_{1}+\delta \theta_{1} \quad \phi_{2}=\phi_{1}+\delta \phi_{1}
$$

Substitui-se $\theta_{1}$ e $\phi_{1}$ em (2.4.9) por $\theta_{2}$ e $\phi_{2}$, resolve-ae 0 sistema e obtóm-se as novas aproximaços $\theta_{3} \oplus \varnothing_{3}$. Ease processo \& repetido até alcançar-se a precisão dese

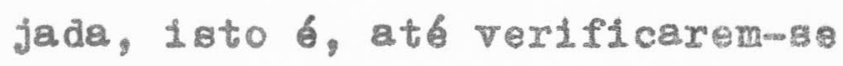

$$
\left|\theta_{1}-\theta_{1-1}\right|<\varepsilon \text { e }\left|\phi_{1}-\emptyset_{1-1}\right|<\delta, \quad \varepsilon>0 \text { \& } \delta>0
$$

A distribuição assintbtica do estimadores dada peIo teorema: (Wald [12]).

Teorema: Considere-se a funço de densidade $f(y ; \vec{\theta})$ em que $\bar{\theta}=\left(\theta_{1}, \theta_{2}, \ldots, \theta_{n}\right)$

Se jam $\hat{\theta}_{1}, \hat{\theta}_{2}, \ldots, \hat{\theta}_{n}$ os estimadorea de máxima veros similhança de $\theta_{1}, \theta_{2}, \ldots, \theta_{n}$, reapectivamente. Entåo a distribuição de probabilidede de $\hat{\theta}$ á asintoticamente

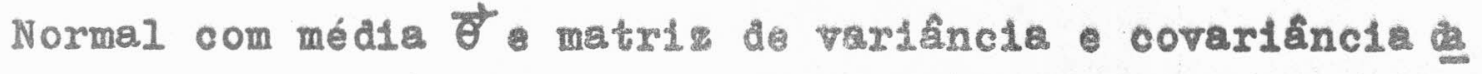
da por: 
eto que

$$
\left\|\sigma_{1 j}(\theta)\right\|=\left\|c_{1 j}(\theta)\right\|^{-1}
$$

$$
c_{1 j}(\theta)=-\mathbb{E}\left(\frac{\partial^{2} \log _{1} I_{1}}{\partial \theta_{1} \partial \theta_{j}}\right)
$$

Observe-se de $(1.4 .5)$ que

$$
E\left(\frac{\partial^{2} I}{\partial \theta_{1} \partial \theta_{j}}\right)=\sum_{1=1}^{k} \frac{-n_{1}}{P_{1} Q_{1}}\left(\frac{\partial P_{1}}{\partial \theta_{1}}\right)\left(\frac{\partial P_{1}}{\partial \theta_{j}}\right)=\frac{\partial^{2} I_{j}}{\partial \theta_{1} \partial \theta_{j}}
$$

que é a expreaвão obt1da em $(1.4 .8)$

Temos asıIm para oョ parâmetros $\theta$ e $\varnothing$ a segulnte metrid de rariâncias e covariância assintótica,

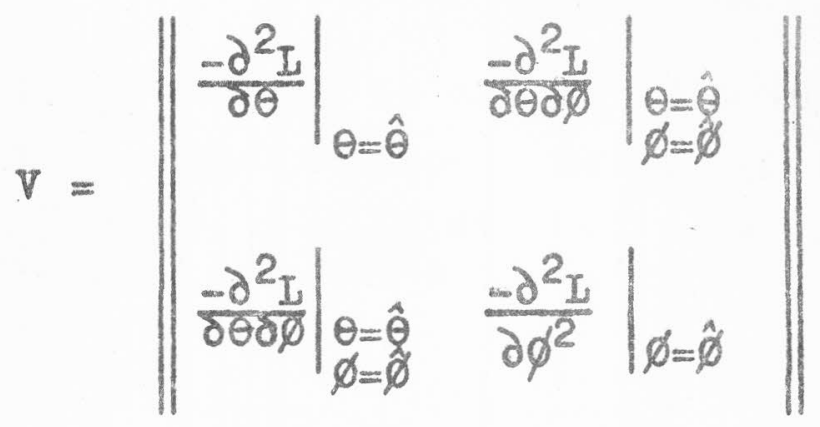

em que $\hat{\theta}$ e $\hat{\emptyset}$ são 8 estimstiva de $\theta$ \& obtids com pre c1são requerida.

\subsection{Estimacão de $\mu$ e $\sigma$}

Os resultados obtidos em $(1.4)$ gão gerals e aplican-se a qualquer distribuicão de tolerânc1a. Suponhs-a gue a distribuiço de tolerância do astluño transtormedo se-

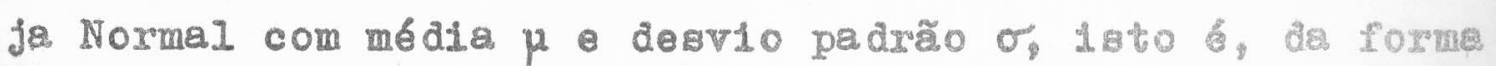
(1.2.3). Já vimor que a relaça entxe o a silmulo trana formado $x$ o problto $Y$ de $P$ \& Inear.

$$
Y=\alpha+\beta x
$$

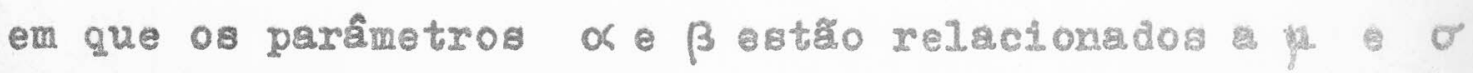
pos

$$
\dot{\mu}=\frac{(5-\alpha)}{\beta} \text { or }=\frac{1}{\beta}
$$


Obtendo eatimativar de $\alpha$ $\beta$, calculam-se Imediata mente de (1.5.1) eatimativar para $\mu$ e $\sigma$.

o probito da proporcão P 6 dado por (1.3.1). Então,

$$
\frac{\partial P}{\partial Y}=\frac{1}{\sqrt{2 \pi}} e^{-\frac{(Y-5)^{2}}{2}}=Z
$$

2 é ordenada da distribuigąo $N(0,1)$ no ponto cuja absc1sga 6 Y 5 .

Temos assim,

$$
\frac{\partial P}{\partial \alpha}=\frac{\partial P}{\partial Y} \cdot \frac{\partial Y}{\partial \alpha}=\mathrm{Z}
$$

중

$$
\frac{\partial P}{\partial \beta}=\frac{\partial P}{\partial Y} \cdot \frac{\partial Y}{\partial \beta}=x z
$$

Suponhamor que $a_{0} e b_{0}$ são aø primeiras aproximaçõอ das estimativas de máxima verossimilhança de $\alpha$ e $\beta$. Subs tituindo $(1.5 .2) \cdot(1.5 .3) \mathrm{em}(1.4 .9)$ temos,

$\left\{\begin{array}{l}\delta a_{0} \sum_{1=1}^{k} \frac{n_{1} z_{1}^{2}}{P_{1} Q_{1}}+\delta b_{0} \sum_{1=1}^{k} \frac{n_{1} z_{1}^{2}}{P_{1} Q_{1}} \cdot x_{1}=\sum_{1=1}^{k} \frac{n_{1} z_{1}^{2}}{P_{1} Q_{1}}\left(\frac{p_{1}-P_{1}}{Z}\right) \\ \delta a_{0} \sum_{1=1}^{k} \frac{n_{1} z_{1}^{2}}{P_{1} Q_{1}} x_{1} \delta_{0} \sum_{1=1}^{k} \frac{n_{1} z_{1}^{2}}{P_{1} Q_{1}} \cdot x_{1}^{2}=\sum_{1=1}^{k} \frac{n_{1} z_{1}^{2}}{P_{1} Q_{1}}\left(\frac{p_{1}-P_{1}}{Z}\right) x_{1}\end{array}\right.$

Observe-se que no sistema (1.5.4) temos as equações normais da regressão ponderada de ( $-\mathrm{P}) \mathrm{z}$ eobre $x$, en que or coeflcientes de ponderação são:

$$
n=\frac{n Z^{2}}{P Q}
$$

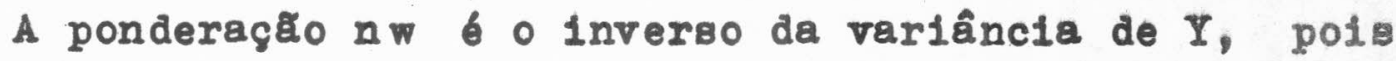
desenvolvendo $p=F(Y)$ pela fórmula de raylor até a derivada de 1 ordem temos

de1 vem

$$
p=F\left(Y_{p}\right)-\left(Y-Y_{p}\right) F^{\prime}\left(Y_{p}\right)
$$

e

$$
\begin{aligned}
& V(p)=\left[I^{\prime}\left(Y_{p}\right)\right]^{2} V(Y) \\
& V(Y)=\frac{P Q}{n Z^{2}}
\end{aligned}
$$


Subst1tu1ndo พ $\mathrm{cm}(1.5 .4)$ vem

$\left\{\begin{array}{l}\delta a_{0} \sum_{1=1}^{k} n_{1} w_{1}+\delta b_{0} \sum_{1=1}^{k} n_{1} w_{1} x_{1}=\sum_{1=1}^{k} n_{1} w_{1}\left(\frac{p_{1}-p_{1}}{z_{1}}\right) \\ \text { Sa, } \sum_{1=1}^{k} n_{1} w_{1} x_{1}+\delta b_{0} \sum_{1=1}^{k} n_{1} w_{1} x_{1}^{2}=\sum_{1=1}^{k} a_{1} w_{1} x_{1}\left(\frac{p_{1}-p_{1}}{z_{1}}\right)\end{array}\right.$

Para rebolvermos o latema (1.5.5) dntroduztremos o probito auriliar que definido por:

$$
y=x+\frac{p-p}{2}-a_{0}+b_{0} x+7: 7=\frac{p-P}{2}
$$

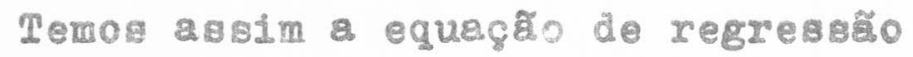

$$
y=a_{1}+b_{1} x
$$

em que

$$
\begin{aligned}
& b_{1}=\frac{\sum_{1=1}^{k} n_{1} w_{1} x_{1}\left(y_{1}-\bar{y}\right)}{\sum_{1=1}^{k} n_{1} w_{1}\left(x_{1}-\bar{x}\right)^{2}} \\
& a_{1}=\bar{y}-b_{1} \bar{x} \\
& \bar{x}=\sum_{1=1}^{k} n_{1} w_{1} x_{1} / \sum_{1=1}^{k} n_{1} w_{1} \quad \bar{y}=\sum_{1=1}^{k} n_{1} w_{1} y_{1} / \sum_{1=1}^{k} n_{1} w_{1}
\end{aligned}
$$

e

$$
b_{2}=b_{0}+\delta b_{0} \quad a_{1}=z_{0}+\delta \varepsilon_{0}
$$

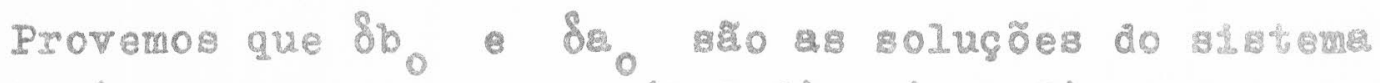
(1.5.5). Substituindo en $(1.5 .6) \cdot(1.5 .7)$ y por $a_{0}+b_{0} x+7$ vem

$$
b_{1}=b_{0}+\frac{\sum_{1=1}^{k} n_{1} w_{1} x_{1}\left(\eta y_{1}-\bar{\eta}\right)}{\sum_{j=1}^{n} w_{1}\left(x_{i}-\bar{x}\right)^{2}} ; \bar{\eta}=\frac{\sum_{1=1}^{k} n_{1} w_{1} \eta_{1}}{\sum_{i=1}^{k} n_{1} w_{1}}
$$




$$
a_{1}=a_{0}+\overline{7}-\bar{z}\left(b_{1}-b_{0}\right)
$$

Temo então

$$
\delta b_{0}=\frac{\sum_{i=1}^{k} w_{1} x_{1}\left(\eta_{1}-\bar{\eta}\right)}{\sum_{i=1}^{k} w_{i}\left(x_{1}-\bar{z}\right)^{2}}
$$

숭

$$
\delta a_{0}=\bar{\eta}-\delta b_{0} \bar{x}
$$

onde $\delta_{0} \odot \delta_{0}$ ač as a

Um novo clelo de caloulos pode ser realizado usando-8s a reta $y=a_{1}+b_{1} x$. 0 processo itexativo gegue ate acan car a preciвão desejade.

Atravén de (1.4.10) obtém-3e a matriz de vaxiâncis covartância as aintót1ca.

$$
V=\left\|\begin{array}{ll}
\sum_{1=1}^{k} n_{1} w_{1} & \sum_{i=1}^{k} n_{1} w_{1} x_{1} \\
\sum_{1=1}^{k} n_{1} w_{1} x_{1} & \sum_{i=1}^{k} n_{1} w_{1} x_{1}^{2}
\end{array}\right\|^{-1}
$$

Temos araim

$$
\begin{aligned}
& v(a)=\frac{\left(\overline{x^{2}}\right)}{S x x} \\
& v(b)=\frac{1}{S x x} \\
& \operatorname{Cov}(a, b)=\frac{-\bar{x}}{S x x} \\
& \text { em que } \quad\left(\overline{x^{2}}\right)=\frac{\sum_{i=1}^{k} n_{1} w_{1} x_{1}^{2}}{\sum_{i=1}^{n} w_{1}} \\
& S x x=\sum_{1=1}^{\sum x} n_{1} w_{1}\left(x_{1}-\bar{x}\right)^{2}
\end{aligned}
$$


Como $\operatorname{cov}(a, b)$ nem sempre nula, trabalharemo com a reta de regreasăo na forma

$$
\Psi=\bar{y}+b(x-\bar{x})
$$

po1s

$$
\operatorname{Cov}(\bar{y}, b)=\operatorname{cov}(a, b)+\bar{x} v(b)=0
$$

Mais ajuda:

$$
V(\bar{y})=V(a)+\bar{x}^{2} V(b)+2 x \operatorname{cov}(a, b)=\frac{1}{\sum_{1=1}^{k} n_{1} w_{1}}
$$

Observação: A. vartâncias $V(\bar{y}) \odot V(b)$ são obtidas somente quando as eatimativa de $\alpha$ e $\beta$ eatão bem próximas das estimativa de méxima verossimilhança.

\subsection{Teste de aderêncle}

Construiremog um testo de hipóteser, a fim de decidir se a di̊tribuiça de tolerância pode oer considerade Normal.

A variável aleatórla $\left(p_{1}-P_{1}\right)$ tem distribulção assin toticamente Normal. con media e variância dada por:

$$
\begin{aligned}
& E\left(p_{1}-p_{1}\right)=0 \\
& V\left(p_{1}-p_{1}\right)=\frac{P_{1} Q_{1}}{n}
\end{aligned}
$$

A eatatiatica

$$
\chi^{2}=\sum_{1=1}^{k} \frac{n_{1}\left(p_{1}-P_{1}\right)^{2}}{P_{1} Q_{1}}
$$

Bob a hipótese de que a distribuição de tolexância é Nor mal, tem distribuição assintoticamente qui-quadrado com ( $k-2)$ graus de 11 berdade, pols dispomos de $\mathbf{k}$ conjuntos de observações 1ndependentes e dua restrições 11neares dadas pelor estimadorea de $\alpha$ e $\beta$.

Através da estatística $\chi^{2}$, temos uma avaliação dos des vios da observaçea com relacão aos valores esperados, 
dando-nog portanto uma medida da aderêncla do modelo pro posto aos dados observados.

A região critica do teate,, 0 nivel de significância $\gamma$, é dada pela região

$$
\left(x^{2}(x-2,1-\gamma) ;+\infty\right)
$$

em que

$\chi^{2}(k-2,1-\gamma)$ é o valor tabelado da distribulção quiquadrado com k-2 grans de l1berdade, tal que

$$
P\left\{\mathrm{X} \leqslant \chi^{2}(k-2,1-\gamma)\right\}=1-\gamma
$$

Como em 1.5 trabalhamos cow $x$ e $y$. façamos algumas transformações em $(1.6 .1)$ de modo a fac1l1tar os calculos Temos que.

$$
w=\frac{\mathrm{z}^{2}}{\mathrm{PQ}}
$$

Então

$$
x^{2}=\sum_{1=1}^{k} n_{1} w_{1}^{\left(\frac{p_{1}-P_{1}}{Z_{1}}\right)^{2}}
$$

Como

$$
Y=I+\frac{p-P}{Z}
$$

vem

$$
\begin{gathered}
x^{2}=\sum_{1=1}^{k} n_{1} w_{1}\left(y_{1}-y_{1}\right)^{2}= \\
=\sum_{1=1}^{k} n_{1} w_{1}\left[y_{1}-\bar{y}-b\left(x_{1}-\bar{x}\right)\right]^{2}= \\
=\sum_{1=1}^{k} n_{1} w_{1}\left(y_{1}-\bar{y}\right)^{2} \frac{-\left[\sum_{1=1}^{k} n_{1} w_{1}\left(y_{1}-\bar{y}\right)\left(x_{1}-\bar{x}\right)\right]^{2}}{\sum_{1=1}^{k} n_{1} w_{1}\left(x_{1}-\bar{x}\right)^{2}}(1.6 .2)
\end{gathered}
$$

Observe-se que o teste de aderêncla realizado através de $(1.6 .2)$ mede os desvios dos probitos observedos em re lação à reta de prob1tos estimada 


\subsection{Intervalos de confianca}

i) Intervalos de confianga para $\alpha$ e $\beta$ Como já vimos em 1.4 os estimadores a e b tem a seguin te distribuiço assintótica.

$$
-N\left(\alpha ; \sqrt{\left(x^{2}\right) / S x x}\right)
$$

e

$$
b \sim N(\beta ; \sqrt{1 / S \cdot x x})
$$

De $(1.7 .1)$ temos que

$$
(a-\alpha ; / \sqrt{V(a)} \sim \mathbb{N}(0 ; 1)
$$

Então um intervalo de $(1-\gamma) \%$ de confiança para $\alpha$ 6

$$
(a-z a \cdot \sqrt{V(a)} ; a+z \gamma \cdot \sqrt{v(a)})
$$

em que z $\gamma$ é valor da abscissa na distribulção $N(0,1)$ ta1 que

$$
P[-z \gamma \leq z \leq z \gamma]=1-\gamma
$$

De $(1.7 .2)$ vem.

$$
(b-\beta) \cdot \operatorname{Sxx}-\mathbb{N}(0,1)
$$

Absim, um Intervalo de $(1-\gamma) \%$ de confiança para $\beta$ é dado por:

$$
\left(b-\frac{z \gamma}{S x x} ; b+\frac{z \gamma}{S x x}\right)
$$

ii) Intervalo de confiança para a reta estimada. A Iim de fazermos previøõe com boa margem de segurança necessitamos conhecer uma região de conflança para a re ta.

Suponhamos que reta ajustada seja dada por

$$
\hat{\mathbf{Y}}=\overline{\mathbf{y}}+b(\mathbf{x}-\overline{\mathbf{x}})
$$

Entåo

$$
\begin{aligned}
V(\hat{X}) & =V(\bar{y})+(x-\bar{x})^{2} V(b)= \\
& =\frac{1}{\sum_{1=1}^{k} n_{1} w_{1}}+\frac{(x-\bar{x})^{2}}{\sum_{1=1}^{k} n_{1} w_{1}\left(x_{1}-\bar{x}\right)^{2}}
\end{aligned}
$$


Temos que $\mathrm{E}(\hat{Y})=\Psi=5+\frac{1}{\sigma}(x-\mu)$

Logo $\frac{\hat{Y}-Y}{\sqrt{V(Y)}} \sim N(0,1)$

Assim um intervalo de $(1-\gamma) \%$ de confiança para $I$ é

$$
\begin{aligned}
& \left(\hat{Y}-z \gamma-\frac{1}{\sum_{1=1}^{k} n_{1} w_{1}}+\frac{\left(x_{1}-\bar{x}\right)^{2}}{\sum_{1=1}^{k} n_{1} w_{1}\left(x_{1}-\bar{x}\right)^{2}}\right] ; \\
& \left.\left.\hat{Y}+z \gamma-\frac{1}{\sum_{1=1}^{k} n_{1} w_{1}}+\frac{\left(x_{1}-\bar{x}\right)^{2}}{\sum_{1=1}^{k} n_{1} w_{1}\left(x_{1}-\bar{x}\right)^{2}}\right]\right)
\end{aligned}
$$

111) Intervalos de confiança para $\mu \in \sigma$

Considere-se a reta $Y=\bar{y}+b(x-\bar{x})$. 0 estimador de $\mu$ a obt1do invertendo-se a reta no ponto $Y=5$. Obtemos

$$
m=\bar{x}+\frac{5-\bar{y}}{b}
$$

Determinaremos primeiramente um intervalo de confian ça para o nivel tranaformado $x$, atravás do teorema de Fieller ( $[7]$, pg. 550)

\section{Teorema de Fieller}

Suponhamos que $\alpha \in \beta$ å̃o do1 parâmetro e

$$
\delta=\frac{\alpha}{\beta}
$$

Sejam a e $\underline{b}$ dols est1madores não-tendenciosos de $\alpha$ - $\beta$ em que $a$ b tem distribuiģa Normal, tal que

$$
V(a)=\sigma^{2} \cdot \nu_{11} ; \quad V(b)=\sigma^{2} \cdot v_{22} ; \operatorname{cov}(a, b)=\sigma^{2} \cdot v_{12}
$$

que $\sigma^{2}$ pode ser desconhecida.

Seja $s^{2}$ um estimador de $\sigma^{2}$ baseado em 1 graus de 11 berdade, tal que 
a) $\frac{e s^{2}}{\sigma^{2}}-x_{1}^{2}$

b) $s^{2}$ é independente de $(a-8 b)$

Então um intervalo de $100(1-\gamma \%)$ para $\delta$ é dado por:

$\left(m+\frac{g}{1-g}\left(m-\frac{\nu_{12}}{\nu_{22}}\right) \pm \frac{t S}{b(1-g)} \sqrt{\left.\nu_{11}-2 m \nu_{12}+m^{2} \nu_{22}-g\left(\nu_{11} \frac{-\nu_{12}^{2}}{\nu_{22}}\right)\right)(1.7 .4)}\right.$

em que $m=\frac{a}{b} \cdot g=\frac{t^{-2} \cdot s^{2} \cdot \varphi_{22}}{b^{2}}$

- t ́ a ordenada da diatribuiça "t" de Student com $P$ graus de Iiberdade.

Quando $g \equiv 0$, o intervalo $(1.7 .4)$ reduz-se a

$$
\left(m \pm \frac{t s}{b} \sqrt{\left.V_{11}-2 m \cdot V_{12}+m^{2} \cdot V_{22}\right)}\right.
$$

as $g \cong 0$ - $\varphi_{12}=0$ temos

$$
\left(m \pm \frac{t .5}{b} \sqrt{\left.v_{11}+m^{2} \cdot v_{22}\right)}\right.
$$

Fazendo a Invergão da reta, a estimativa por ponto de x 6 :

$$
\hat{x}=\bar{x}+\frac{\hat{y}-\bar{y}}{b} \longrightarrow \hat{x}-\bar{x}=\frac{\hat{y}-\bar{y}}{b}
$$

Colocando $a=\hat{Y}-\bar{y}_{3} m=a / b$ é um eatimador para $(x-\bar{x})$, satisfazendo o teorema de Fleller, pola

a) $\underline{a}$ e b são estimadores não tendenciosos de $(Y-\bar{y})$ e $\beta$, respectivamente e tem distribuisao asint6ticamente Normal.

b) $v(a)=v(\bar{y})=\frac{1}{\sum_{1=1}^{k} n_{1} w_{1}}$

$V(b)=\frac{1}{S x x}$

$\operatorname{Cov}(a, b)=0$ 
c) $\sigma^{2}$ á conhecido í igual a 1

Então um intervalo de $(1-\gamma) \%$ de confiança para $x$ é

$$
\left(x+\frac{B}{1-8}(x-\bar{x}) \pm \frac{z \gamma}{b(1-g)} \sqrt{\frac{1-B}{\sum_{1=1}^{k} n_{1} w_{1}}+\frac{(x-\bar{x})^{2}}{S x x}}\right)(1.7 .5)
$$

$B=\frac{(z \gamma)^{2}}{b^{2} \cdot 5 x x}$

De $(1.7 .3)$ e (1.7.5) obtemos o seguinte intervalo de $(1-\gamma) \%$ para $\mu$,

$$
\left(m+\frac{g}{1-8}(m-\bar{x}) \pm \frac{z \gamma}{\delta(1-g)} \sqrt{\frac{1-8}{\sum_{1=1}^{k} n_{1} w_{1}}+\frac{(x-\bar{x})^{2}}{S x x}}\right)
$$

Um intervalo de confiança para $\sigma$ é encontrado atravéa da relação $\sigma=1 / \beta$. Ao nível de confiança $(1-\gamma) \%$ temos o intervalo

$$
\left(\frac{S x x}{b . S x x+2 \gamma} ; \frac{S x x}{b \cdot S x x-2 \gamma}\right)
$$

1.8. Comparação entre duas distribuicões de tolerância

Frequentemente no estudo de distribuições de tolerân cia tem-se como interesse testar se doís estímulos têm a mesma potência.

Sejam A B dois estimulos que foram aplicados em di versos niveis a lotes de unidades experimentais. Suponhamos que a partir dos dados observados obtivemos as seguintes retas de regressão

$$
\begin{aligned}
& Y_{A}=\bar{y}_{A}+b_{A}\left(x-\bar{x}_{A}\right) \\
& Y_{B}=\bar{y}_{B}+b_{B}\left(x-\bar{x}_{B}\right)
\end{aligned}
$$

Ora, para testarmos se potênça dos estímulos A 


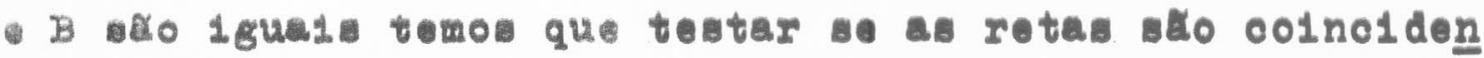
tes. Para tal, primelramente testaremos se os coeficientes angulares das retas de probltos a a 1 guals. Acelta eg a hipbtene testaremos ae os coeflalentes 11nearea alo iguais.

1) Teste de paralel1smo

Queremos testar as hipbteaes

$\begin{cases}H_{0}: & \beta_{A}=\beta_{B} \\ H_{I}: & \beta_{A} \neq \beta_{B}\end{cases}$

Sabemos que

$$
\left(b_{A}-b_{B}\right)-N\left(\beta_{A}-\beta_{B} ; \sqrt{\frac{1}{S_{x x}^{A}}+\frac{1}{B}}\right)
$$

Sob $\mathrm{H}_{0}$ :

$$
\left(b_{A}-b_{B}\right)-N\left(0 ; \sqrt{\frac{1}{S_{x x}}+\frac{1}{B}}\right)
$$

Então a eatatiatica

$$
Q=\frac{\left(b_{A}-b_{B}\right)^{2}}{\frac{1}{S}+\frac{1}{S_{x x}}}
$$

tem distribuição aสaintot1camente qui-quadrado com 1 grau de liberdade.

A região critica do teste a nivel de significancia de $\gamma \%$ é

$$
\left(\chi^{2}(1,1-\gamma) ;+\infty\right)
$$

Suponhamos que aceitamos a hipótese $\mathrm{H}_{0}$. Então as dua reta de regreasăo podem ser considerada parale 1as. Uma estimativa comum para o coeficiente angular $\beta_{A}=\beta_{B}=\beta$ é obtida pela média ponderada dos coeflo1entes angulares $b_{A}$ e $b_{B}$, usando os valores reciprocos das variânclas como pesor, 1sto é,

$$
\bar{b}=\frac{s_{x x}^{A} b_{A}+s_{x x}^{B} b_{B}}{S x x+S x x}=\frac{s_{x y}^{A}+s_{x y}^{B}}{S x x+s x x} \text { ex que }
$$

$$
S x y=\sum_{1=1}^{k}\left(y_{1}-\bar{y}\right)\left(x_{1}-\bar{x}\right)
$$


A estimativa $\bar{b}$ fol obtida através do método dos mínimos quadrados.

Se ja

$$
\begin{gathered}
R=\sum_{i=1}^{k_{A}}\left[y_{j}^{A}-\bar{y}_{A}-b\left(x_{i}^{A}-\bar{x}\right)\right]^{2}+ \\
+\sum_{i=1}^{k_{B}}\left[y_{i}^{B}-\bar{y}_{B}-b\left(x_{i}^{B}-\bar{x}\right)\right]^{2} \\
\text { o estimador } \vec{b} \text { é solução da equagão } \\
\frac{\partial R}{\partial b}=0
\end{gathered}
$$

$\overline{\mathrm{b}}$ tem distribuição assintoticamente Normal com média $\beta$ e variância

$$
V(b)=\frac{1}{S x x+S x x}
$$

As equações para as retas de regressão teóricas com o mesmo coeficiente angular são:

$$
\begin{aligned}
& Y_{A}=\bar{y}_{A}+\beta\left(x-\bar{x}_{A}\right) \\
& Y_{B}=\bar{y}_{B}+\beta\left(x-\bar{x}_{B}\right)
\end{aligned}
$$

Passaremos agora ao

ii) Tegte de igualdade do coeficiente lineax Testaremos as hipóteses $H_{0}: \bar{y}_{A}-\beta \cdot \bar{x}_{A}=\bar{y}_{B}-\beta \cdot \bar{x}_{B}$ ou $\beta=\frac{\bar{y}_{A}-\bar{y}_{B}}{\bar{x}_{A}-\bar{x}_{B}}$ $\mathrm{H}_{I}: \quad \beta \neq\left(\overline{\mathrm{y}}_{\mathrm{A}}-\overline{\mathrm{y}}_{\mathrm{B}}\right) /\left(\overline{\mathrm{x}}_{\mathrm{A}}-\overline{\mathrm{x}}_{\mathrm{B}}\right)$

A estatistica

$$
\hat{b}=\frac{\hat{\bar{y}}_{A}-\hat{\bar{y}}_{B}}{\bar{x}_{A}-\bar{x}_{B}}
$$

é um estimador não tendencioso de $\beta$

$\hat{b}$ representa o coeficiente angular da reta que passa pelos pontos $\left(\bar{x}_{A}, \overline{\bar{y}}_{A}\right)$ e $\left(\bar{x}_{B}, \hat{\bar{y}}_{B}\right) \cdot \bar{b}$ e $\hat{b}$ são esto - 
casticamente independentes porque $\bar{b}$ fol calculada através da variação dentro da amostra e $\hat{b}$, através da variação entre as amoatra.. Se as retas de regressão té́r1cas aão 1dênticas, então a variação entre $\hat{b}$ e $\vec{b}$ será pu ramente aleatória.

Como a variância de $\hat{b}$ é Igual a

$y(\hat{b})=\frac{1}{\left(\bar{x}_{A}-\bar{x}_{B}\right)^{2}}\left(\frac{1}{\sum_{1=1}^{k} n_{1}^{A} w_{1}}+\frac{1}{\sum_{1=1}^{k} n_{1}^{B} w_{1}}\right)$

temos

$v(\hat{b}-\bar{b})=\frac{1}{\left(\bar{x}_{A}-\bar{x}_{B}\right)^{2}}\left(\frac{1}{\sum_{1=1}^{k} n_{1}^{A} w_{1}}+\frac{1}{\sum_{1=1}^{k} n_{1}^{B} w_{1}}-\frac{1}{S A x+S_{x x}^{B}}\right.$

Testar $H_{0}$ equivale a testar se as duas retas são ldên ticas. Então sob Ho,

$$
v=(\hat{b}-\bar{b}) / \sqrt{v(\hat{b}-\bar{b})}
$$

tem aistribuição assintoticamente Normal com média 0 e variância 1 .

Através da estatistica I podemos testar as hipóteses propostas. 
2. DISTRIBUICKO DE TOLERANCIA DA MISTURA DE DOIS ESTIMULOS INDEPENDENTES

2.1. Determinação da distribuicão de tolerância de uma mistura a partir da distribuicão de tolerância de seus componentes.

Daremos primeiramente algumas definições e notações que serão utilizadas através deste capitulo.

Conslderaremos que as unldades experimenta1s são compostas de diversos sistemas.

Definicão 1: Denomina-se mistura de estímulos, a do1s ou mais estimulos que são aplicados simultaneamente a unidades experimentais.

Denotaremos a mistura de dols estímulos A e B por (A; B), o nivel transformado do estimulo $A$ por $x_{1}$, o $n \underline{f}$ vel transformado do estimulo $\mathrm{B}$ por $\mathrm{x}_{2}$ e os niveis da mistura por $\left(x_{1}, x_{2}\right)$

Definição 2: Dizemos que dols estimulos tem ações inde pendentes, (são independentes) se eles agem sobre sistemas distintos e a ação de um estímulo não interfere na ação do outro [1]

Pela definição 2 vemos que se aplicarmos um nível $\left(x_{1}, x_{2}\right)$ da mistura de estímulos independentes $(A, B)$ a uma unidade experimental ela irá responder ao estimulo se a sua tolerância a $A$ é inferior a $x_{1}$ ou se a sua to lerância a $B$ é inferior a $x_{2}$.

A distribuição de tolerância de uma mistura de estí mulos independentes $(A ; B)$ não é unicamente determinada através das distribuições de tolerâncias de $A$ e $B$ em separado. E necessário conhecer também o quanto a tolerância a A está correlacionada à tolerância a B na população em que se está trabalhando. Observe-se que se a tolerância ao estimulo A é completa positivamen te correlacionada à tolerância do estimulo $B$, então uma 
unidade experimental muito tolerante ao eatímulo A será também muito tolerante ao etímulo B. Se a tolerância ao eatímulo A é completa o negativamente correlacionada a tolerância do estimulo B, uma unidade experimental muito tolerante a A será pouco tolerante a B e vice-versa. Se a correlação é incompleta e o individuo é muito tolerante ao estímilo $A$, existirá uma probabilidade, maior ou menor, de acordo com o grau de correlagão, da unidade ser muito tolerante ao estimulo B.

Estudaremos primeiramente, a distribuicão da mistura, nos caroa em que o coeficiente de correlaça, $\rho$, é igual a $-I, I$ e 0 , utilizando as distribuiçõe de tolerêncla de A e B, segundo Plackett e Hewlett [10].

Sejam $p_{1}$ e $p_{2}$ as proporços de resposta provocadas, respectivamente, pelos niveis $x_{1}$ de $A x_{2}$ de $B$, quando aplicados a lotes de unidader experimentaic. Seja p a proporç̃o de reaposta obtida quando aplica o nivel $\left(x_{1}, x_{2}\right)$ da mistura de estímulos independentes $(A, B)$ a u lote de unidader experimentais. Então,

a) Se $p=1$, 0 estimulo A Irá provocar resposta em $p_{1} \%$ de unidades experimental menos resirtentes a esse estimulo e $\mathrm{p}_{2} \%$ da unidader experimentala menos resistentes a B Irão reaponder a este estimulo.

Se $p_{1} \geq p_{2}$, as $p_{2} \%$ unidades experimentalo menos reais tentes ao estímulo $\mathrm{B}$ pertencem ds $\mathrm{p}_{1} \%$ undades experimentai menos tolerantes a eatimulo A. Temobo mes mo raciocínio quando $p_{2} \geqslant p_{1}$. Portanto a relaço exio tente entre $p_{1} p_{1}$ e $p_{2}$ 6́

$$
\begin{array}{lll}
p=p_{1} & \text { \& } & p_{1} \geqslant p_{2} \\
p=p_{2} & \text { se } & p_{2} \geqslant p_{1}
\end{array}
$$

b) Se $\rho=0$, os estimulos $\mathrm{A}$ e B ẵo estocasticamente Inde pendenter, de modo que probabilidade de resposta d̀ mistura ́́ dada pors

$$
p=p_{1}+p_{2}-p_{1} p_{2}
$$


Então $p \geqslant p_{1}$ - $p \geq p_{2}$, Iogo, a mistura $(A, B)$ de est1mulos independentes \& mais potente do que os eatimuI0\& $A \subset B$.

c) Se $p=-1$ e $p_{1}+p_{2} \leqslant 1$, o conjunto das und dades experimentais não tolerantes $80 \mathrm{n}$ Ivel $\mathrm{x}_{1}$ de $\mathrm{A}$ - conjun to das unidades experimentais não tolerantes ao nivel $x_{2}$ de $B$ вão mutuamente exclusivos. Assim

$$
p=p_{1}+p_{2} \quad \text { se } \quad p_{1}+p_{2} \leqslant 1
$$

Se $\rho=-1$ e $p_{1}+p_{2} \geq 1$, todas as unidades experimentais Irão reaponder ao eatlmulo $\left(x_{1}, x_{2}\right)$ de $(A, B)$, então

$$
\mathrm{p}=1 \quad \text { se } \quad \mathrm{p}_{1}+\mathrm{p}_{2} \geq 1
$$

Logo, a potência da mistura é superior à potência dos estímulog $A$ e B.

Para o caso em que a correlação entre a tolerâncla 6 Incompleta, BI1⿴ [3] propôa o aeguinte modelo,

$$
p=p_{1}+p_{2}\left(1-p_{1}\right)(1-p)
$$

que é incompleto porque aó é vålido para $0 \leq \rho \leq 1$.

Hewlett e Plackett [10] pesquisaram sobre a possibil1 dade de representar $p$ como um polinômio em $\rho$ cujos coeficientes fossem combinaços IIneares de $p_{1}$ e $p_{2}$ e con cluíram que seria 1mpossivel. Tornou-se então necessária a construção de um modelo que envolvesse $p$ e que fog se válida para $-1 \leqslant \rho \leqslant 1$. Deata forma Hewlett o Plackett

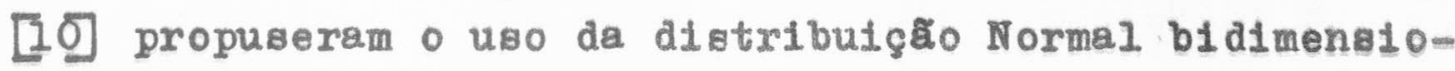
nal. 
2.2. Ueo da dLatribudea Normal como d1stribuigão de tolerâncis de misturs de dols estimulos independentes

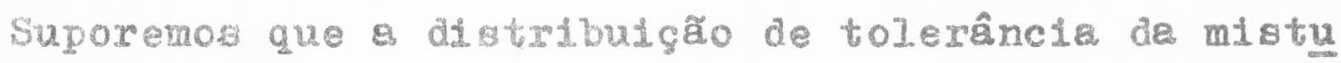
re de dols etimulos indeperdentse $(\mathrm{A}, \mathrm{B})$ \& normal bidi w. menstonal, 1 to 6, wua funga do denaidade é dada pox.

$$
\begin{aligned}
& p\left(x_{1}, \pi_{2}\right)=\frac{1}{2 \pi \sigma_{1} \sigma_{2} \sqrt{1-p^{2}}} \operatorname{erp}\left[\frac { - 1 } { 2 ( 1 - p ^ { 2 } ) } \left[\left(\frac{x_{1}-\mu_{1}}{\sigma_{1}}\right)^{2}+\left(\frac{x_{2}-p_{2}}{\sigma_{2}}\right)^{2}\right.\right. \\
& \left.+\frac{2 p\left(x_{1}-p_{1}\right)\left(x_{2}-p_{2}\right)}{\sigma_{1}}\right]
\end{aligned}
$$

ex que

$-\infty<x_{1}<+\infty, \quad-\infty<x_{2}<4+\infty \theta \sigma_{1}, \sigma_{2}, \mu_{1}, \mu_{2}, p$ в râmetros thà çue,

$\sigma_{1} \rightarrow 0 ; c_{2} \times 0 ;-\infty<\mu_{1}<+\infty ;-\infty<p_{2}<+\infty e-1<p<1$

A pextro da dado quanta:s os parâmetros $\mu_{1}, \sigma_{1}, \mu_{2}$

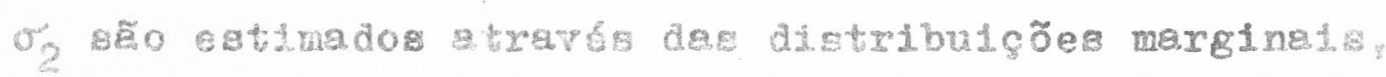
Msendo o wétodo de probitos descrtto ao cepltulo 2. Sum ponhamos que tenhamos oneortredo an estimetivas: $P_{1}^{0}$. $\mathrm{H}^{0}$ o. $\sigma_{2}^{0}$ a pazts da amostxas cu jo tamanho geja tal gue

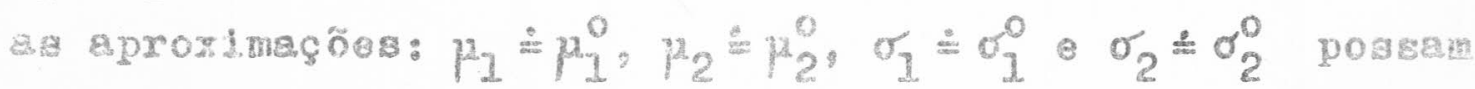

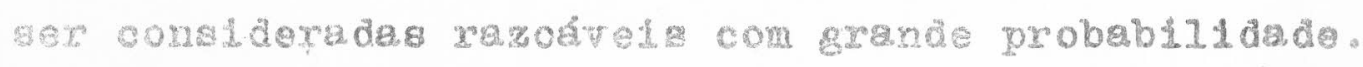

Far

$$
z_{1}=\frac{x_{1}-\mu_{1}^{0}}{\sigma_{1}^{0}} \quad z_{2}=\frac{x_{2}-\mu_{2}^{0}}{\sigma_{2}^{0}}
$$

obtomos am $(2.2 .1)$

$f\left(z_{1}, z_{2}\right)=\frac{1}{2 \pi \sqrt{1-p^{2}}} \exp \left[\frac{-1}{2\left(1-p^{2}\right)}\left(z_{1}^{2}+z_{2}^{2}-2 p z_{1} z_{2}\right)\right](2.2 .2$ entä : aplearmos un nLFel $\left(x_{1}, x_{2}\right)$ de mistura $(A, B)$ de 
estímulos independentes a uma unidade experimental, a pro babilidade dela responder ao estímulo é

$$
\begin{aligned}
P & =\int_{-\infty}^{z_{1}} \int_{z_{2}}^{+\infty} f(u, v) d u d v+\int_{z_{I}}^{+\infty} \int_{-\infty}^{z_{2}} f(u, v) d u d v+ \\
& +\int_{-\infty}^{z_{1}} \int_{-\infty}^{z_{2}} f(u, v) d u d v
\end{aligned}
$$

A probabilidade de não resposta ao estímulo é

$$
Q=1-P=
$$$$
=\int_{z_{1}}^{+\infty} \int_{z_{2}}^{+\infty} \frac{1}{2 \pi \sqrt{1-\rho^{2}}} \exp \left[\frac{-1}{2\left(1-\rho^{2}\right)}\left(u^{2}+v^{2}-2 \rho u v\right)\right] d u d v(2.2 .3)
$$

$\mathrm{Na}$ figura 1 temos no plano a secça da distra buição Normal bidimensional por planos paralelos aos eixos dos $u$ e $v$, que se cortam no ponto $T$.

- volume sob a normal bidsmensional que eatá sobre o setor KTS representa $(2.2 .3)$.

Quando $\rho=1$ a distribulgão Normal bidimenajonal reduz-se a uma distribuição Normal unidimenaional, em que as massas de probabilidade encontram-se distri buidas sobre a reta $u=v$ da figura $I$.

A figura $2 a$ mostra a dítribuiça Normal que se encontra sobre a reta $u=v$ da figura 1. Nessa mesma figura vemos que $z_{2}>z_{1}$ portanto $p_{2}>p_{1}$ e $p=p_{2}$. Da mesma forma, se $z_{1} \geq_{2}$ texímos $p=p_{1}$. Esвea resultados foram encontrados em (2.1.1) e (2.1.2) .

Quando $\rho=-1$, a distribujção Normal bjdimensional re- 


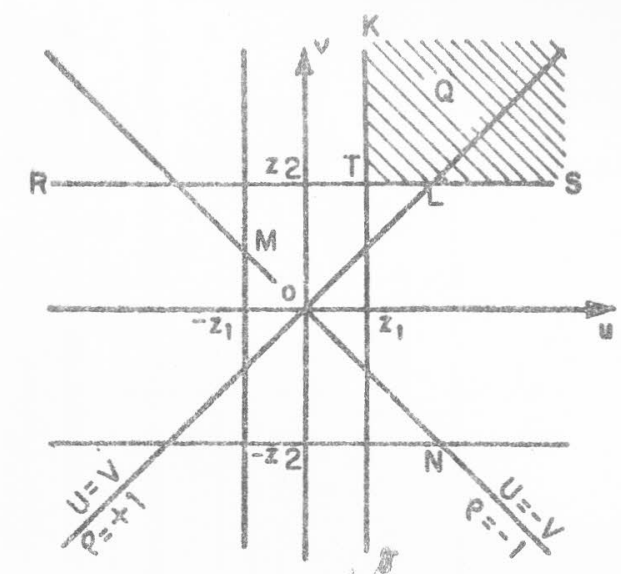

Fio. 1

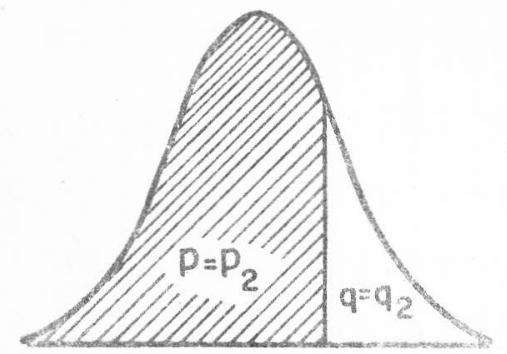

Fig. 2 을

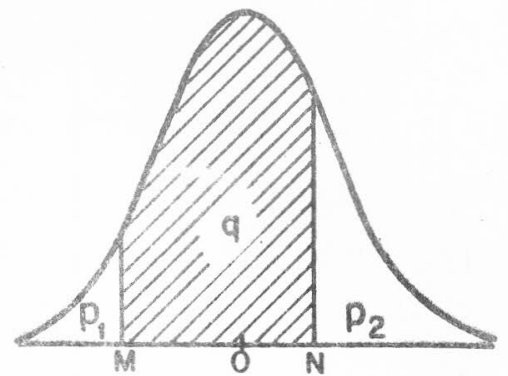

Fig 2 b

duz-se a uma distribujģo Noxmal unidimensional, cuja masa de probabilidade encontra-se alatribulda sobre are ta $u=-\nabla$ da figura 1. Esa distribuiço tem a forma da Iigura 2b. Para o núvel $\left(z_{1} s_{2}\right)$ temo que $p=1$, como pow de aer visto na figura 1, pole $p_{1}+p_{2} \geq 1$. Para o nirel $\left(-z_{1},-z_{2}\right)$ temos que $p=p_{1}+p_{2}$ como pode ser $v 1$ to to as flgura 1. Temos assim (2.1.4) e (2.1.5)

Quando $p=0$ temos

$$
q=\int_{z_{1}}^{+\infty} \frac{e^{-u^{2} / 2}}{\sqrt{2 \pi}} d u \int_{z_{2}}^{+\infty} \frac{e^{-v^{2} / 2}}{\sqrt{2 \pi}} d v=q_{1} q_{2}
$$

portanto

$$
p=p_{1}+p_{2}-p_{1} p_{2}
$$




\subsection{Estimacão de $P$}

Suponhamos que k niveis da mistura $(A, B)$ de estimu10 independentes são aplicados a k lotes de unidades ex perimentais de tamanhos $n_{1}, n_{2}, \ldots, n_{k}$. 0 estimador de máxima verossimilhança de $p$ é, de acordo com (1.4.2), a solução da equação

$\frac{\partial I}{\partial \rho}=\sum_{1=1}^{k} \frac{n_{1}\left(p_{1}-P_{1}\right)}{P_{1} Q_{1}} \frac{\partial P_{1}}{\partial \rho}=\sum_{1=1}^{k} \frac{n_{1}\left(q_{1}-Q_{1}\right)}{P_{1} Q_{1}} \frac{\partial Q_{1}}{\partial p}=0$

em que $Q$ é dado por (2.2.3)

A função $\partial L / \partial \rho$ só assume valores reais para $p \in(-1 ; I)$ e é decrescente nesse intervalo pols $\partial^{2} L / \partial \rho^{2}<0$ de acordo com (2.3.3). Suponhamos que $\rho^{*}$ se ja o parâmetro verdadeiro da população. Então para um mesmo nível $\left(x_{1}, x_{2}\right)$, quando $\rho<\rho^{*}-\delta \quad(\delta>0) \quad P$ aumenta $\log 0 Q$ diminui e $\partial I / \partial \rho$ é positivo. Para $\rho>p^{*}+\delta$ P diminui e por tanto $Q$ aumenta fazendo com que $\partial L / \partial \rho<0$. Portanto no intervalo $\left(\rho^{*}-\delta ; \rho^{*}+\delta\right)$ a função $\partial L / \partial \rho$. tem sempre uma raiz e ela única.

Se ja

$$
\psi\left(z_{1}, z_{2}, \rho\right)=\frac{1}{2 \pi \sqrt{1-p^{2}}} \exp \left\{\frac{-1}{2\left(1-p^{2}\right)}\left(z_{1}^{2}+z_{2}^{2}-2 p z_{1} z_{2}\right)\right]
$$

Então,

$$
\frac{\partial Q_{1}}{\partial \rho}=\int_{z_{1}^{1}}^{+\infty} \int_{z_{2}^{1}}^{+\infty} \frac{\partial}{\partial \rho} \psi\left(z_{1}, z_{2}, \rho\right) d z_{2} d z_{1}
$$

Mas,

$$
\begin{gathered}
\frac{\partial \psi\left(z_{1}, z_{2}, \rho\right)}{\partial \rho}=\frac{\partial^{2} \psi\left(z_{1}, z_{2}, \rho\right)}{\partial z_{1} \partial z_{2}}= \\
=\frac{1}{2 \pi\left(1-\rho^{2}\right)^{3 / 2}} \exp \left[-\frac{1}{2\left(1-\rho^{2}\right)}\left(z_{1}^{2}+z_{2}^{2}-2 \rho z_{1} z_{2}\right)\right] .
\end{gathered}
$$


$-29-$

$$
\cdot\left[\rho+\frac{z_{1} z_{2}-\rho\left(z_{1}^{2}+z_{2}^{2}-\rho z_{1} z_{2}\right)}{\left(1-e^{2}\right)}\right]
$$

Então

$$
\begin{aligned}
\frac{\partial Q_{1}}{\partial \rho} & =\int_{z_{1}^{1}}^{+\infty} \int_{z_{2}^{1}}^{+\infty} \frac{\partial^{2} \psi\left(z_{1}, z_{2}, \rho\right)}{\partial z_{1} \partial z_{2}} d z_{1} d z_{2}= \\
& =\int_{z_{2}^{1}}^{+\infty} \frac{\partial}{\partial z_{2}}\left[\int_{z_{1}^{1}}^{+\infty} \frac{\partial \psi\left(z_{1}, z_{2}, \rho\right)}{\partial z_{1}} d z_{1}\right] d z_{2}= \\
& =\int_{z_{2}^{1}}^{+\infty}-\frac{\partial \psi\left(z_{1}^{1}, z_{2}, \rho\right)}{\partial z_{2}} d z_{2}=\psi\left(z_{1}^{1}, z_{2}^{1}, \rho\right)
\end{aligned}
$$

Iogo, temos em (2.3.1)

$$
\frac{\partial I}{\partial \rho}=\sum_{1=1}^{k} n_{1} \psi\left(z_{1}^{1}, z_{2}^{i}, \rho\right) \frac{\left(q_{1}-Q_{1}\right)}{P_{i} Q_{1}}=0
$$

A equação $(2.3 .2)$ é não Ilnear e diflcilmente explí citável. Portanto, determinaremos uma estimativa de $\rho$ atravé do método iterativo de Newton.

Seja $r_{0}$ uma estimativa inicial de $\rho$. Atravéa do mé todo de Newton determinamos sucessivamente ar eatimativas $r_{1}, r_{2}, \ldots, r_{k}, r_{k+1}, \ldots$ de $p$ que convergem para a estimativa de máxima verosalmilhança de $\rho$. A est1mativa $x_{k}$ da $k$-ésima 1 teração é dada pela expresaão

$$
r_{k+1}=r_{k}-\frac{[\partial L / \partial \rho] \rho=r_{k}}{\left[\partial^{2} L / \partial \rho^{2}\right] \rho=r_{k}}
$$


em que

$$
\frac{\partial^{2} I}{\partial \rho^{2}}=-\sum_{1=1}^{k} \frac{n_{1}\left[\psi\left(z_{1}^{1}, z_{2}^{1}, \rho\right)\right]^{2}}{P_{1}^{Q_{1}}}
$$

de acordo $\operatorname{com}(1.4 .6)$

Encerra-se o processo quando a precisão desejada é al cançada.

A rapidez da convergêncla do método de Newton depende do valor inicial $r_{0}$. Quanto mals proximo $r_{0}$ eativer da raiz da equação, mais ráplda a convergêncla. Para determinar $x_{0}$ podemos utilizar o método usado por Hewlett e Plackett [8] para eatimar $\rho$, que consiste em cal

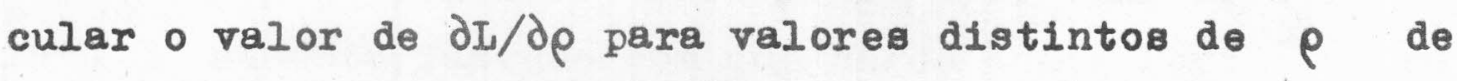
tal forma que tenhamos $\partial \mathrm{I} / \partial \rho<0$ e $\partial \mathrm{I} / \partial \rho>0$. Obtemoa então $r_{0}$ atravéa de Interpolação numérica. Podemos também obter $r_{0}$ fazendo uma pesquisa de sinal ou simplesmente colocar sempre $r_{0}=0$ porque para este valor inicial a convergência do processo á garantida devido à forma de $\partial I / \partial \rho$ (gráflco 1 ) como verificaremorno próximo capitulo.

Como já vimos em (1.4) o estimador $\underline{r}$ de $\rho$ tem dietribuição asaintoticamente Normal com média $\rho$ variância.

$$
v(r)=\left[\frac{\partial^{2} u}{\partial \rho^{2}}\right]_{\rho=r}^{-1}
$$

Usando a distribuição assint6t1ca de $r$ podemos obter um intervalo de confiança para $\rho$. 
GRÁFICO I

GRÁFICO DA FUNÇÃO $\frac{\partial L}{\partial \ell}$ PARA OS DADOS DA TABELA 2-0

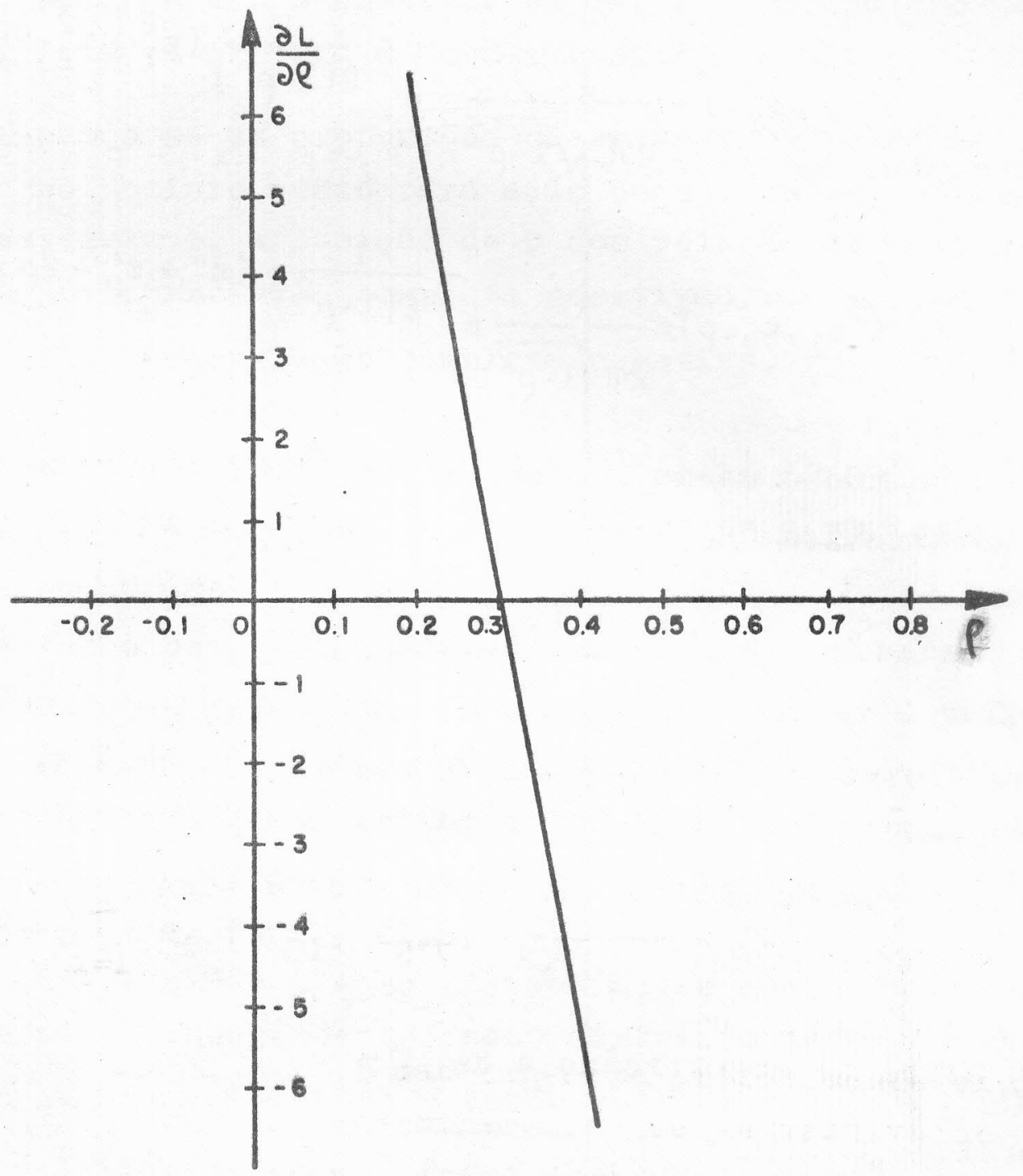




\subsection{Teste de aderência}

Determinada uma eatimativa de $\rho$, interessa-nos decí dir se a dịtribuição de tolerância encontrada adere bem aos dados observados. Isto é, Intereasa-nos testar as hí pótesea

$$
\begin{aligned}
& H_{0}: f\left(z_{1}, z_{2} \rho\right)=\frac{1}{2 \pi \sqrt{1-\rho^{2}}} e^{-\frac{1}{2\left(1-\rho^{2}\right)}\left(z_{1}^{2}+z_{2}^{2}-2 \rho z_{1} z_{2}\right)} \\
& H_{1}: f\left(z_{1}, z_{2}, \rho\right) \neq \frac{1}{2 \pi \sqrt{1-\rho^{2}}} e^{-\frac{1}{2\left(1-\rho^{2}\right)}\left(z_{1}^{2}+z_{2}^{2}-2 \rho z_{1} z_{2}\right)}
\end{aligned}
$$

Usando o mesmo raciocinio de (1.6) temos que sob $\mathrm{H}_{\mathrm{o}}$ a estatistica

$$
x^{2}=\sum_{i=1}^{k} \frac{n_{1}\left(p_{1}-P_{1}\right)^{2}}{P_{1} Q_{1}}
$$

tem distribuição qui-quadrado com $(k-1)$ graus de liberda de porque $\rho$ fol eat1mado a partir da. k observações.

A região crítica do teate é dada por

$$
\left(\chi^{2}(k-1,1-\gamma) ;+\infty\right)
$$

ao nível de gígnificância de $\gamma \%$

2.5. Roteiro para a análise da mistura de dois estímulos independentes

a) Fazemos observações de $k_{1}$ e $k_{2}$ doses dos estimulos $A$ e $B$, respectivamente e de $k_{3}$ doses da mistura $(A, B)$ de estímulos independentes. Temos portanto, $k=k_{1}+k_{2}+k_{3}$ lotes experimentais.

b) Calculam-se as retas de regressão para os estímulos $A$ a $B$ usando o método de probitos apresentado no ca 
pítulo 1. Eatimamos então a partir dos parâmetros das retas as médias $\mu_{1}$ e $\mu_{2}$ e as variâncias $\sigma_{1}$ e $\sigma_{2}$.

c) Procedemos a um teste qui-quadrado para testar a norma Iidade da distribuição de tolerância de A e B. Se a normalidade de pelo menos ume das marginals fôr rejeitada, abandonamos a hipótese de que a distribuição de tolexância da mistura é Normal bidimensional.

d) Verificamos se as proporções observadas de resposta p estão no domínio certo para ação de estímulos independentes, isto é, a posição de p com relação ao máximo de $\left(p_{1}, p_{2}\right)$ (correlaçä́ completa positiva) e o mínimo de $\left(p_{1}+p_{2} ; I\right)$ (correlação completa negativa)

1) Se todos os $p$ estão entre o máx $\left(p_{1} ; p_{2}\right)$ e o mín. $\left(p_{1}+p_{2} ; 1\right)$, estima-se $p$ e procede-se a um teste qui-quadrado.

2) Se todos os $p$ estão acima do mín. $\left(p_{1}+p_{2} ; I\right)$, procede-se a um teste $x^{2} \operatorname{com} \rho=-1$. Se $\chi^{2}$ não é significante então $\rho=-1$, caso contrário a ação dos estímulos quase certamente não é independente.

3) Se todos os $p$ estão abalxo do máx $\left(p_{1} ; p_{2}\right)$, procedemos a um teste $\chi^{2}$ com $\rho=+1$. Se $\chi^{2}$ é significativo então a ação dos estímulos quase certamente não é independente, caso contrário temos $\rho=+1$.

4) Se alguns valorea de $p$ estão entre min $\left(p_{1}+p_{2} ; I\right)$ e máx $\left(p_{1}, p_{2}\right)$, estimamos $p$ e fazemos um teste $\chi^{2}$. Se $\chi^{2}$ não fôr significativo então não há evidências pâ ra dizer que as ações dos eatímulos não aล̃o indepen dentes, caso contrário consideramo-1as não-independentes.

\subsection{Alternativas para a estimação de $\mu_{1}, \mu_{2}, \sigma_{1}, \sigma_{2}$ e}

De acordo com (1.4.2) os estimadores de máxima verossi milhança de $\mu_{1}, \mu_{2}, \sigma_{1}, \sigma_{2} e$ são as soluções do aistema: 


$$
\left\{\begin{array}{l}
\frac{\partial I}{\partial \mu_{1}}=\sum_{1=1}^{k} \frac{n_{1}\left(q_{1}-Q_{1}\right)}{P_{1} Q_{1}} \frac{\partial Q_{1}}{\partial \mu_{1}}=0 \\
\frac{\partial L}{\partial \mu_{2}}=\sum_{i=1}^{k} \frac{n_{1}\left(q_{1}-Q_{1}\right)}{P_{1} Q_{1}} \frac{\partial Q_{1}}{\partial \mu_{2}}=0 \\
\frac{\partial L}{\partial \sigma_{1}}=\sum_{1=1}^{k} \frac{n_{1}\left(q_{1}-Q_{1}\right)}{P_{1} Q_{1}} \frac{\partial Q_{i}}{\partial \sigma_{1}}=0 \\
\frac{\partial I}{\partial \sigma_{2}}=\sum_{1=1}^{k} \frac{n_{1}\left(q_{1}-Q_{1}\right)}{P_{1} Q_{1}} \frac{\partial Q_{1}}{\partial \sigma_{2}}=0 \\
\frac{\partial I}{\partial \rho}=\sum_{i=1}^{k} \frac{n_{1}\left(q_{1}-Q_{1}\right.}{P_{1} Q_{1}} \frac{\partial Q_{1}}{\partial \rho}=0
\end{array}\right.
$$

A solução desse sistema pode ser obtida através do mé todo iterativo de Newton. Desenvolvendo as equações de (2.6.1) em séries de Taylor até as derivadas de 1 a ordem e considerando que em (1.4.8) obtivemos

$$
\frac{\partial^{2} L}{\partial \theta \partial \phi}=-\sum_{i=1}^{k} \frac{n_{1}}{P_{1} Q_{1}}\left(\frac{\partial P_{1}}{\partial \theta}\right)\left(\frac{\partial P_{1}}{\partial \phi}\right)=-\sum_{1=1}^{k} \frac{n_{1}}{P_{1} Q_{1}}\left(\frac{\partial Q_{1}}{\partial \theta}\right)\left(\frac{\partial Q_{1}}{\partial \phi}\right)
$$

Chegamos ao sistema:

$$
\begin{aligned}
& \sum_{i=1}^{k} \frac{n_{i}\left(q_{1}-Q_{1}\right)}{P_{1} Q_{i}} \frac{\partial Q_{1}}{\partial \mu_{1}}=\delta_{1} \sum_{1=1}^{k} \frac{n_{1}}{P_{1} Q_{1}}\left(\frac{\partial Q_{1}}{\partial \mu_{1}}\right)^{2}+ \\
& +\delta_{2} \sum_{i=1}^{k} \frac{n_{i}}{P_{1} Q_{1}}\left(\frac{\partial Q_{i}}{\partial \mu_{1}}\right)\left(\frac{\partial Q_{1}}{\partial \mu_{2}}\right)+\delta_{3} \sum_{i=1}^{k} \frac{n_{1}}{P_{1} Q_{1}}\left(\frac{\partial Q_{i}}{\partial \mu_{1}}\right)\left(\frac{\partial Q_{1}}{\partial \sigma_{1}}\right)+ \\
& +\delta_{4} \sum_{i=1}^{k} \frac{n_{1}}{P_{1} Q_{1}}\left(\frac{\partial Q_{1}}{\partial \mu_{1}}\right)\left(\frac{\partial Q_{1}}{\partial \sigma_{2}}\right)+\delta_{5} \sum_{1=1}^{k} \frac{n_{1}}{P_{1} Q_{1}}\left(\frac{\partial Q_{i}}{\partial \mu_{1}}\right)\left(\frac{\partial Q_{1}}{\partial \rho}\right)
\end{aligned}
$$


$-35-$

$$
\begin{aligned}
& \sum_{i=1}^{K} \frac{n_{i}\left(q_{1}-Q_{1}\right)}{P_{1} Q_{1}}\left(\frac{\partial Q_{1}}{\partial \mu_{2}}\right)=\delta_{1} \sum_{i=1}^{K} \frac{n_{1}}{P_{1} Q_{1}}\left(\frac{\partial Q_{1}}{\partial \mu_{2}}\right)\left(\frac{\partial Q_{1}}{\partial \mu_{1}}\right)+ \\
& +\delta_{2} \sum_{i=1}^{k} \frac{n_{1}}{P_{i} Q_{1}}\left(\frac{\partial Q_{1}}{\partial \mu_{2}}\right)^{2}+\delta_{3} \sum_{1=1}^{k} \frac{n_{1}}{P_{1} Q_{1}}\left(\frac{\partial Q_{1}}{\partial \mu_{2}}\right)\left(\frac{\partial Q_{1}}{\partial \sigma_{1}}\right)+ \\
& +\delta_{4} \sum_{i=1}^{k} \frac{n_{1}}{P_{1} Q_{1}}\left(\frac{\partial Q_{1}}{\partial \mu_{2}}\right)\left(\frac{\partial Q_{1}}{\partial \sigma_{2}}\right)+\delta_{5} \sum_{i=1}^{k} \frac{n_{1}}{P_{1} Q_{1}}\left(\frac{\partial Q_{1}}{\partial \mu_{2}}\right)\left(\frac{\partial Q_{1}}{\partial \rho}\right) \\
& \sum_{i=1}^{K} \frac{n_{1}\left(q_{1}-Q_{1}\right)}{P_{1} Q_{i}}\left(\frac{\partial Q_{1}}{\partial \sigma_{1}}\right)=\delta_{1} \sum_{i=1}^{K} \frac{n_{i}}{P_{1} Q_{i}}\left(\frac{\partial Q_{i}}{\partial \sigma_{1}}\right)\left(\frac{\partial Q_{i}}{\partial p_{1}}\right)+ \\
& +\delta_{2} \sum_{i=1}^{k} \frac{n_{1}}{P_{1} Q_{1}}\left(\frac{\partial Q_{1}}{\partial \sigma_{1}}\right)\left(\frac{\partial Q_{1}}{\partial \mu_{2}}\right)+\delta_{3} \sum_{1=1}^{k} \frac{n_{i}}{P_{1} Q_{1}}\left(\frac{\partial Q_{i}}{\partial \sigma_{1}}\right)^{2}+ \\
& +\delta_{4} \sum_{i=1}^{k} \frac{n_{i}}{P_{1} Q_{i}}\left(\frac{\partial Q_{i}}{\partial \sigma_{1}}\right)\left(\frac{\partial Q_{i}}{\partial \sigma_{2}}\right)+\delta_{5} \sum_{i=1}^{k} \frac{n_{i}}{P_{i} Q_{1}}\left(\frac{\partial Q_{1}}{\partial \sigma_{1}}\right)\left(\frac{\partial Q_{i}}{\partial \rho}\right. \\
& \sum_{i=1}^{k} \frac{n_{i}\left(q_{i}-Q_{i}\right)}{P_{i} Q_{i}}\left(\frac{\partial Q_{i}}{\partial \sigma_{2}}\right)=\delta_{1} \sum_{i=1}^{k} \frac{n_{i}}{P_{i} Q_{i}}\left(\frac{\partial Q_{i}}{\partial \sigma_{2}}\right)\left(\frac{\partial Q_{i}}{\partial \mu_{1}}\right)+ \\
& +\delta_{2} \sum_{i=1}^{K} \frac{n_{i}}{P_{1} Q_{i}}\left(\frac{\partial Q_{1}}{\partial \sigma_{2}}\right)\left(\frac{\partial Q_{1}}{\partial \mu_{2}}\right)+\delta_{3} \sum_{i=1}^{K} \frac{n_{i}}{P_{1} Q_{1}}\left(\frac{\partial Q_{1}}{\partial \sigma_{2}}\right)\left(\frac{\partial Q_{1}}{\partial \sigma_{1}}\right)+ \\
& +\delta_{4} \sum_{i=1}^{k} \frac{n_{i}}{P_{i} Q_{i}}\left(\frac{\partial Q_{i}}{\partial \sigma_{2}}\right)^{2}+\delta_{5} \sum_{i=1}^{k} \frac{n_{1}}{P_{i} Q_{i}}\left(\frac{\partial Q_{i}}{\partial \sigma_{2}}\right)\left(\frac{\partial Q_{1}}{\partial \rho}\right) \\
& \sum_{i=1}^{k} \frac{n_{i}\left(q_{1}-Q_{1}\right)}{P_{1} Q_{1}}\left(\frac{\partial Q_{i}}{\partial \rho}\right)=\delta_{1} \sum_{1=1}^{k} \frac{n_{1}}{P_{1} Q_{1}}\left(\frac{\partial Q_{i}}{\partial \rho}\right)\left(\frac{\partial Q_{1}}{\partial \mu_{1}}\right)+ \\
& +\delta_{2} \sum_{i=1}^{k} \frac{n_{1}}{P_{i} Q_{1}}\left(\frac{\partial Q_{1}}{\partial \rho}\right)\left(\frac{\partial Q_{1}}{\partial \mu_{2}}\right)+\delta_{3} \sum_{1=1}^{k} \frac{n_{1}}{P_{1} Q_{1}}\left(\frac{\partial Q_{1}}{\partial \rho}\right)\left(\frac{\partial Q_{1}}{\partial \sigma_{1}}\right)+
\end{aligned}
$$


$+\delta_{4} \sum_{1=1}^{k} \frac{n_{1}}{P_{1} Q_{1}}\left(\frac{\partial Q_{1}}{\partial \rho}\right)\left(\frac{\partial Q_{1}}{\partial \sigma_{2}}\right)+\delta_{5} \sum_{1=1}^{k} \frac{n_{1}}{P_{1} Q_{1}}\left(\frac{\partial Q_{1}}{\partial \rho}\right)^{2}$

Se $\mu_{1}^{0}, \mu_{2}^{0}, \sigma_{1}^{0}, \sigma_{2}^{0}$ e $\rho^{0}$ são estimativas iniciais dé $\mu_{1}, \mu_{2}, \sigma_{1}, \sigma_{2}$ e respectivamente, usamos esses valores para resolver o sistema (2.6.2) obtendo assim as eatimativas refinadar,

$$
\begin{aligned}
& \mu_{1}^{1}=\mu_{1}^{0}+\delta_{1} ; \quad \mu_{2}^{1}=\mu_{2}^{0}+\delta_{2} ; \quad \sigma_{1}^{1}=\sigma_{1}^{0}+\delta_{3} \\
& \sigma_{2}^{1}=\sigma_{2}^{0}+\delta_{4} ; \quad \rho^{1}=\rho^{0}+\delta_{5}
\end{aligned}
$$

Com essas novas estimativas resolvemos novamente(2.6.2) obtendo novas aproximações das estimativas de máxima ve rosaimilhança. Esse processo se repete até obtermos a precisão dese jada.

Outro processo de resolver o sistema (2.6.1) foi proposto por Moore-Zelgler [9]. Consiste em fazer uma equivalência entre as equações de máxima-verossimilhança de $(2.6 .1)$ e as equações norma1s de uma regressão não 11 near.

Supondo-ge independência e variânciag não necessariamente 1gua1s, 0 modelo de regressão não linear pode ser apresentado da seguinte forma:

$$
y_{1}=g\left(z_{1}, \beta\right)+e_{1} \quad 1=1,2, \ldots, m
$$

em que

$$
\beta=\left(\beta_{1}, \beta_{2}, \ldots, \beta_{n}\right)
$$

o estimador de mínimos quadrados de $\beta$ é obtido minimi zando-se

$$
Q=\sum_{1=1}^{m} w_{1}\left[y_{1}-g\left(z_{1}, \beta\right)\right]^{2}
$$

em que $W_{1}$ é o peso associado à 1-ésima observação e é ge ralmente o reciproco da variância de $e_{1}$.

A condição necessária de mínimo é: 
$\frac{\partial Q}{\partial \beta_{l}}=-\sum_{1=1}^{m} w_{1}\left[y_{1}-g\left(z_{1}, \beta\right)\right] \frac{\partial g\left(z_{1}, \beta\right)}{\partial \beta_{l}}=0 \quad(2.6 .3)$ $\ell=1,2, \ldots, n$

Podemor entro fazer a aegutnte equivalência entre $(2.6,3) \bullet(2.6,1)$

$$
\begin{gathered}
{\left[a_{1}(\theta) \leftrightarrow\left(z_{1}, \beta\right)\right] ;\left[a_{1}=y_{1}\right] ;\left[w_{1} \leftrightarrow n_{1} / p_{1} q_{1}\right]} \\
\theta=\left(\ddot{r}_{1}, \mu_{2}, \sigma_{1}, \sigma_{2}, \rho\right)
\end{gathered}
$$

Deata forma $(2.6,1)$ pode ser conaiderada como 0 siste

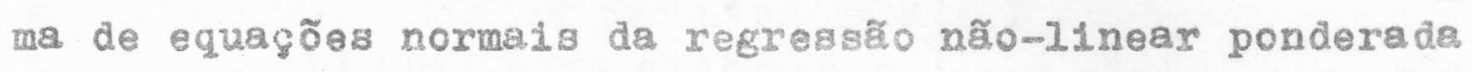

$$
\begin{aligned}
q_{1}=Q_{1}(\theta)+\varepsilon_{1}, \quad 1 & =1,2, \ldots, k \\
E\left(\varepsilon_{1}\right) & =0 ; V\left(\varepsilon_{1}\right)=n_{1} / P_{1} Q_{1}
\end{aligned}
$$

e pode sex resolvido como tal.

$\theta$ pode sex estimado através do método da linearização.

A eatimação dos cinco parâmetros através desses dola métodos apresentados, envolve o cálculo de $\partial Q / \partial \mu_{1}$, $\partial Q / \partial \mu_{2}, \partial Q / \partial \sigma_{1}, \partial Q / \partial \sigma_{2}$, que bastante elaborado. 


\section{APLICACRO}

\subsection{Simulação}

Simulanos em computador cinco experiências que consistiram em:

i) Aplicar seis níveis do estimulo A, com distribuição de tolerância $N(1,8 ; 0,5)$ em 6 lotes de unidades experimentais cada um com 20 unidades e verificar após o número de unidades que responderam a cada ní vel.

11) Realizar a mesma experiência do 1 tem (i) com o estí mulo B com distribuigão de tolerância $N(2,5 ; 0,5)$.

1ii) Realizar trề vezes a seguinte experiência:

Aplicar a 32 lotes, cada um com 40 unidades experimentais, niveis da mistura de estímulos independentes $(A ; B)$ com distribuição de tolerância Normal bi dimensional com parâmetros.
a) $\mu_{1}=1,8 ; \quad \mu_{2}=2,5 ; \quad \sigma_{1}=\sigma_{2}=0,5 ; \quad \rho=-0,8$
b) $\mu_{1}=1,8 ; \quad \mu_{2}=2,5 ; \quad \sigma_{1}=\sigma_{2}=0,5 ; \quad \rho=0,1$
c) $\mu_{1}=1,8 ; \quad \mu_{2}=2,5 ; \quad \sigma_{1}=\sigma_{2}=0,5 ; \quad \rho=0,6$

e verificar aṕ́s o número de unidades experimentais que responderam a cada nivel.

Os resultados das simulações das experiências (i) e

(ii) encontram-se na Tabela 1 e os resultados das simula ções das experiências descritas em (iii), na Tabela 2.

3.2. Estimacão de $\mu_{1}, \mu_{2}, \sigma_{1}, \sigma_{2} \underline{e} \rho$

A partir dos resultados de simulação, estimamos os parâmetros da distrıbuição Normal bidimensional.

Usamos o método 1terativo descrito em (1.5) para es timar os parâmetros $\mu_{1}, \mu_{2}, \sigma_{1} \in \sigma_{2}$. Aplicando o método 
dos minimos quadrados aos valores da Tabela 1 encontra mos as estimativas iniciais

$a_{0}=2,207$ e $b_{0}=1,456$ para os parâmetros da reta de probitos do eatímalo A $\theta$

$a_{0}=1,422$ e $b_{0}=1,477$ para os parâmetros da reta de probitos do estimulo B.

Com esses valores iniciamos o processo iterativo e o critério de parada adotado foi:

$\left|b_{1}-b_{1-1}\right| \leq 0,01$ e $\left|a_{1}-a_{1-1}\right| \leq 0,01$

Os cálculos foram feitos em computador e encontramos as seguintes retas de probitos de A e B, respectivamente,

$$
Y_{1}=1,949+1,671 x_{1}
$$
e

$$
Y_{2}=0,949+1,648 x_{2}
$$

Temos, então, de $(1.5 .1)$ as seguintes estimativas para $\mu_{1}, \mu_{2}, \sigma_{1}$ e $\sigma_{2}$.

$$
\begin{array}{ll}
\hat{\mu}_{1}=\frac{5-1,949}{1,671}=1,83 ; & \hat{\sigma}_{1}=\frac{1}{1,671}=0,598 \\
\hat{\mu}_{2}=\frac{5-0,949}{1,648}=2,46 ; & \hat{\sigma}_{2}=\frac{1}{1,648}=0,607
\end{array}
$$

Antes de estimarmos $\rho$ fizemos testes de aderência de acordo com (1.6.2) para verificarmos a normalidade das distribuições de tolerância das marginaia A e B.

Encontramos para a reta (3.2.1) o seguinte valor do qui-quadrado

$$
\text { Qui }=4,159
$$

Como o valor tabelado da distribuição qui-quadrado com quatro graus de liberdade, ao nivel de significância de $5 \%$ é

$$
\chi^{2}(4 ; 0,95)=9,49
$$

aceitamos a hipótere de normalidade da distribuição de tô 
Lerânc1e de A, pola Qu1 $<x^{2}(4,0,95)$

Para a reta $(3.2 .2)$ encontramos Qui $=3,814$ então, como Qui $<\chi^{2}(4 ; 0,95)$ aceitamos a hipótese de normalidade da distribuição de tolerância de B.

Como aceitamos a hipótese de que as distribuições de tolerância de ambos estímulos são Normaia, passamos à e日 timação de $\rho$.

A estimativa de $\rho$ foi obtida através do procesøo apre sentado em (2.3). O valor inicial do processo fol encon irado fazendo uma pesquisa de sinal. Como dL/op é sempre decrescente (gráfico 1), a pesquisa fol feita da seguinte maneira:

Calculamos $\partial I / \partial \rho$ para valores de $\rho$ a partir de $-0,9$, de décimo em décimo, até encontrarmos $\partial \mathrm{L} / \partial \rho<0$. Esse valor de $\rho$ foi tomado como valor inicial do processo ite rativo de Newton.

Encontramos as seguintes estimativas para os dados da Tabela 2.
(s) $\hat{\rho}=-0,86$
(b) $\hat{\rho}=-0,05$
(c) $\hat{\rho}=0,3$

\subsection{Teste de Aderência}

Realizamos após, um teate de aderência, usando a di̊ tribuição qui-quadrado.

0 valorea calculados através da fórmula (2.4.1) pa ra os dados da Tabela 2 foram:

a) Qui $=25,027$

b) Qui $=30,446$

c) Qui $=32,219$

O valor tabelado da distribuição Qui-quadrado com 31. graus de liberdade, ao nível de aignificância de $5 \%$ ć

$$
x^{2}(31 ; 0,95) \cong 43,8
$$

Temos portanto que Qui $<\chi^{2}(31 ; 0,95)$ para (a). (b) 
e (c); aceitamos a hipótese de que os dados da Tabela 2, provêm de populações com distribuição Normal bidimensio nal.

\subsection{Convergência do processo iterativo de Newton}

Devido à forma da função (gráfico l)

$$
\frac{\partial I}{\partial \rho}=\sum_{i=1}^{k} \frac{n_{i}\left(q_{i}-Q_{i}\right)}{Q_{i} P_{1}} \psi_{i}
$$

a convergência do méivå de Newton é bem rápida para a de terminação da sua raiz.

Para verificar a rapidez da convergência iniciamos o processo iterativo de Newton com $\rho_{0}=0$. A estimativa $\hat{p}=-0,86$ para os dados da tabela $2-a$ foi obtida apos 6 iterações. Como o processo de obtenção do valor inicial descrito em (3.2) essa mesma estimativa foi obtida após 3 iterações.

A estimativa $\hat{\rho}=-0,05$ para os dados da tabela $2-b$ foi obtida ap6s 6 iterações, usando $\rho_{0}=-0,98$, e com 0 processo descrito em (3.2) a convergência foi obtida aṕs duas iterações.

\section{Tabela 1}

Resultados da simulação de amostras de estímulos com distribuicão de tolerância $N(\mu ; \sigma)$

\begin{tabular}{|c|c|c|c|c|c|}
\hline \multicolumn{3}{|c|}{$X_{I}: N(1,8 ; 0,5)$} & \multicolumn{3}{|c|}{$\mathrm{X}_{2}: \mathrm{N}(2,5 ; 0,5)$} \\
\hline NÍvel & $\begin{array}{l}\text { Tamanho } \\
\text { do lote }\end{array}$ & $\begin{array}{r}\text { Número de } \\
\text { respostas }\end{array}$ & Nivel & $\begin{array}{l}\text { Tamanho } \\
\text { do lote }\end{array}$ & $\begin{array}{l}\text { Numero de } \\
\text { respostas }\end{array}$ \\
\hline 0,8 & 20 & 0 & 1,4 & 20 & 0 \\
\hline 1,2 & 20 & 4 & 1,8 & 20 & 5 \\
\hline 1,5 & 20 & 6 & 2,2 & 20 & 5 \\
\hline 1,9 & 20 & 13 & 2,7 & 20 & 13 \\
\hline 2,2 & 20 & 12 & 3,0 & 20 & 17 \\
\hline 2,5 & 20 & 18 & 3,3 & 20 & 18 \\
\hline
\end{tabular}




\section{Tabela 2}

Resultados da simulação de amostras de misturas de dois estimulos independentes com distribuicão Normal Bidimensional com $\mu_{1}=1,8 ; \mu_{2} \equiv 2,5 ; \quad \sigma_{1} \equiv \sigma_{2}=0,5 \mathrm{e}$

\section{$\rho$ de acordo com a tabela}

\begin{tabular}{|c|c|c|c|c|c|c|c|c|c|}
\hline \multirow{2}{*}{ NLveI } & \multirow{2}{*}{$\begin{array}{c}\text { Tama } \\
\text { nho } \\
\text { do } \\
\text { Lote }\end{array}$} & \multicolumn{3}{|c|}{6} & \multirow{2}{*}{ Nivel } & \multirow{2}{*}{$\begin{array}{c}\text { Tama } \\
\text { nho } \\
\text { do } \\
\text { lote }\end{array}$} & \multicolumn{3}{|c|}{$p$} \\
\hline & & $\begin{array}{r}(a) \\
-0,8\end{array}$ & $\begin{array}{l}(b) \\
0,1\end{array}$ & $\begin{array}{l}(c) \\
0,6\end{array}$ & & & $\begin{array}{l}(a) \\
-0,8\end{array}$ & $\begin{array}{l}(b) \\
0,1\end{array}$ & $\begin{array}{l}(c) \\
0,6\end{array}$ \\
\hline$(0,8 ; 1,4)$ & 40 & 1 & 1 & 1 & $(1,9 ; 2,2)$ & 40 & 28 & 24 & 21 \\
\hline$(0,8 ; 1,8)$ & 40 & 5 & 5 & 4 & $(1,9 ; 2,7)$ & 40 & 39. & 32 & 30 \\
\hline$(0,8 ; 1,7)$ & 40 & 25 & 24 & 23 & $(1,9 ; 3,0)$ & 40 & 40 & 38 & 37 \\
\hline$(0,8 ; 3,0)$ & 40 & 37 & 36 & 35 & $(1,9 ; 3,3)$ & 40 & 40 & 39 & 39 \\
\hline$(0,8 ; 3,3)$ & 40 & 38 & 38 & 37 & $(2,2 ; 1,4)$ & 40 & Ju & 30 & 30 \\
\hline$(1,2: 1,4)$ & 40 & 3 & 3 & 3 & $(2,2 ; 1,8)$ & 40 & 33 & 32 & 30 \\
\hline$(1,2 ; 2,2)$ & 40 & 17 & 16 & 15 & $(2,2 ; 2,2)$ & 40 & 38 & 38 & 35 \\
\hline$(1,2 ; 3,0)$ & 40 & 37 & 36 & 36 & $(2,2 ; 2,7)$ & 40 & 40 & 37 & 34 \\
\hline$(1,5 ; 1,4)$ & 40 & 10 & 10 & 9 & $(2,2 ; 3,0)$ & 40 & 40 & 37 & 36 \\
\hline$(1,5 ; 1,8)$ & 40 & 19 & 17 & 17 & $(2,2 ; 3,3)$ & 40 & 40 & 40 & 40 \\
\hline$(1,5 ; 2,2)$ & 40 & 21. & 18 & 16 & $(2,2 ; 1,4)$ & 40 & 37 & 37 & 37 \\
\hline$(1,5 ; 2,7)$ & 40 & 33 & 24 & 23 & $(2,5 ; 1,8)$ & 40 & 36 & 36 & 36 \\
\hline$(1,5 ; 3,0)$ & 40 & 38 & 36 & 36 & $(2,5 ; 2 ; 2)$ & 40 & 39 & 37 & 37 \\
\hline$(1,5 ; 3,3)$ & 40 & 37 & 36 & 36 & $(2,5 ; 2,7)$ & 40 & 40 & 39 & 37 \\
\hline$(1,9 ; 1,4)$ & 40 & 26 & 25 & 25 & $(2,5 ; 3,0)$ & 40 & 40 & 39 & 38 \\
\hline$(1,9 ; 1,8)$ & 40 & 28 & 27 & 27 & $(2,5 ; 3,3)$ & 40 & 40 & 40 & 40 \\
\hline
\end{tabular}




\section{APENDICE}

\section{PROGRAMA I}

\section{Finalidade do programa}

O programa calcula estimativas para os parâmetros $\alpha$ e $\beta$ da reta de probitos através do método iterativo de New ton.

\section{Diretrizes do Programa}

O programa foi escrito em linguagem FORTRAN IV, do computador IBM 1130 que é uma variação do FORTRAN IV (ASA) padronizado internacionalmente e aceito pela malor parte dos computadores.

A listagem do programa encontra-se na figura 3 e é composta de uma função (Q GAUS) e um programa principal.

A função QGAUS calcula o valor da integral

$$
\int_{x}^{+\infty} \frac{1}{\sqrt{2 \pi}} e^{-\frac{x^{2}}{2}} d x \quad x \in \mathbb{R}
$$

A precisão dessa função é da ordem de $5 \times 10^{-8}$

\section{Dados necessários}

A figura 4 apresenta uma tabela dos simbolos utilizados para referenciar os dados.

Na figura 5 apresentamos o formato em que devem ser perfurados os cartões de dados, bem como a ordem em que devem ser colocados esses cartões no maço de cartões que seré levado ao computador.

\section{Resultados fornecidos}

Na figura 6 são apresentados os resultados fornecidos 
pelo computador.

Além das estímativas $A$ \& $B$ também aão fornecidos valo res adiciona1: para realizar-ae um terte de aderêncla, calculax varianciab e tntervalo de confiança.

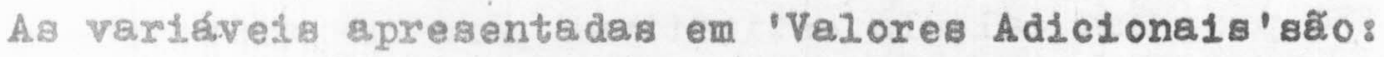

QUI = valor do quí-quadrado

SNW $=\sum_{1=1}^{k} n_{1} w_{1}$

SNWX $=\sum_{1=1}^{k} n_{1} w_{1} x_{1}$

SNWX2 $=\sum_{i=1}^{k} n_{1} w_{1} x_{1}^{2}$

SNWY $=\sum_{i=1}^{k} n_{i} w_{1} y_{i}$

SNWY $2=\sum_{i=1}^{k} n_{i} w_{i} y_{i}^{2}$

SNWXY $=\sum_{1=1}^{k} n_{1} w_{1} x_{1} y_{1}$

Precisão do programa

A preclsão de $A e^{-B}$ é fixada pelo uøuário. Na I1stagem apresentada a precLsão para A $B$ são Iguais ou superiores a um centéaimo.

\section{Capacidade do programa}

o programa apxesentado na 11stagen da figura 3 admite até no máximo 100 nive1s. Entretanto, ume I1geira modificaça no comando DIMENSION pode aumentar a capacidade do programa até o méximo permitido pela momória do computador. 


\section{Figura 3}

* Listagem do deck * *

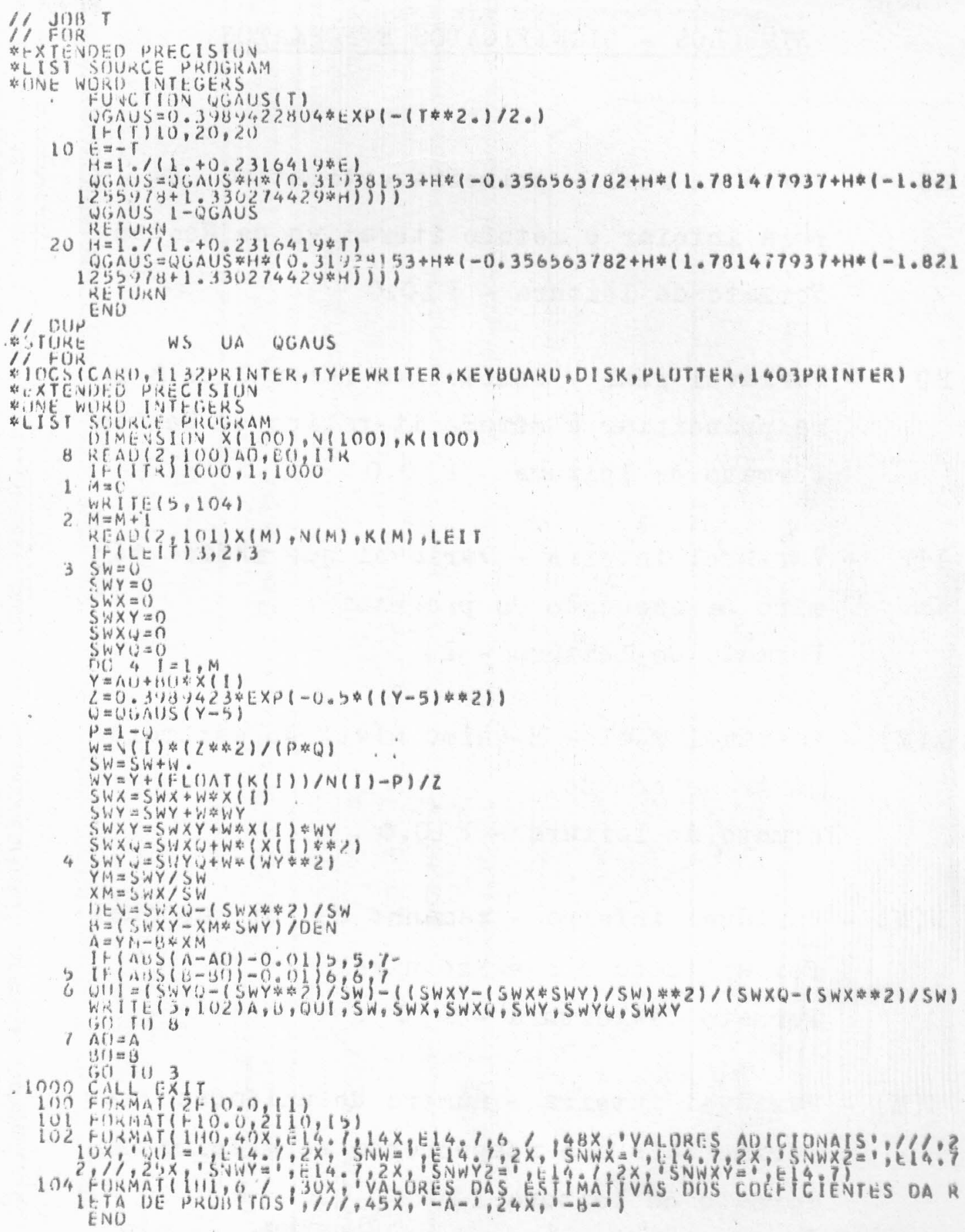




\section{Fisura 4}

\section{SIMBOLOS - SIGNIFICADOS E FORMATOS}

AO - Variável real - primeira eatimativa de $\alpha$ para iniciar o método iterativo de Newton. Formato de leitura - F10.0

BO - Variável real - estimativa inicial de $\beta$ pa ra principiar o método iterativo de Newton Formato de leitura - F10.0

ITR - Variável inteira - variável que indica tér mino da execução do programa

Formato de leitura - I I

$X(M)$ - Variável real - M-ésimo nivel do estimulo (já transformado)

Formato de ieitura - F10.0

$N(M)$ - Variável inteira - tamanho do lote ao qual foi aplicado o m-ésimo nível

Formato de leitura - I 10

$K(M)$ - Variável inteira - número de unidades experimentáis que responderam ao m-ésimo nível Formato de leitura I 10

IEIT - Variável inteira - variável que indica o término da leitura dos niveis. Formato de leitura I 5 
Fig. 5

DISPOSIC̨ÃO DOS DADOS DE ENTRADA DO PROGRAMA I

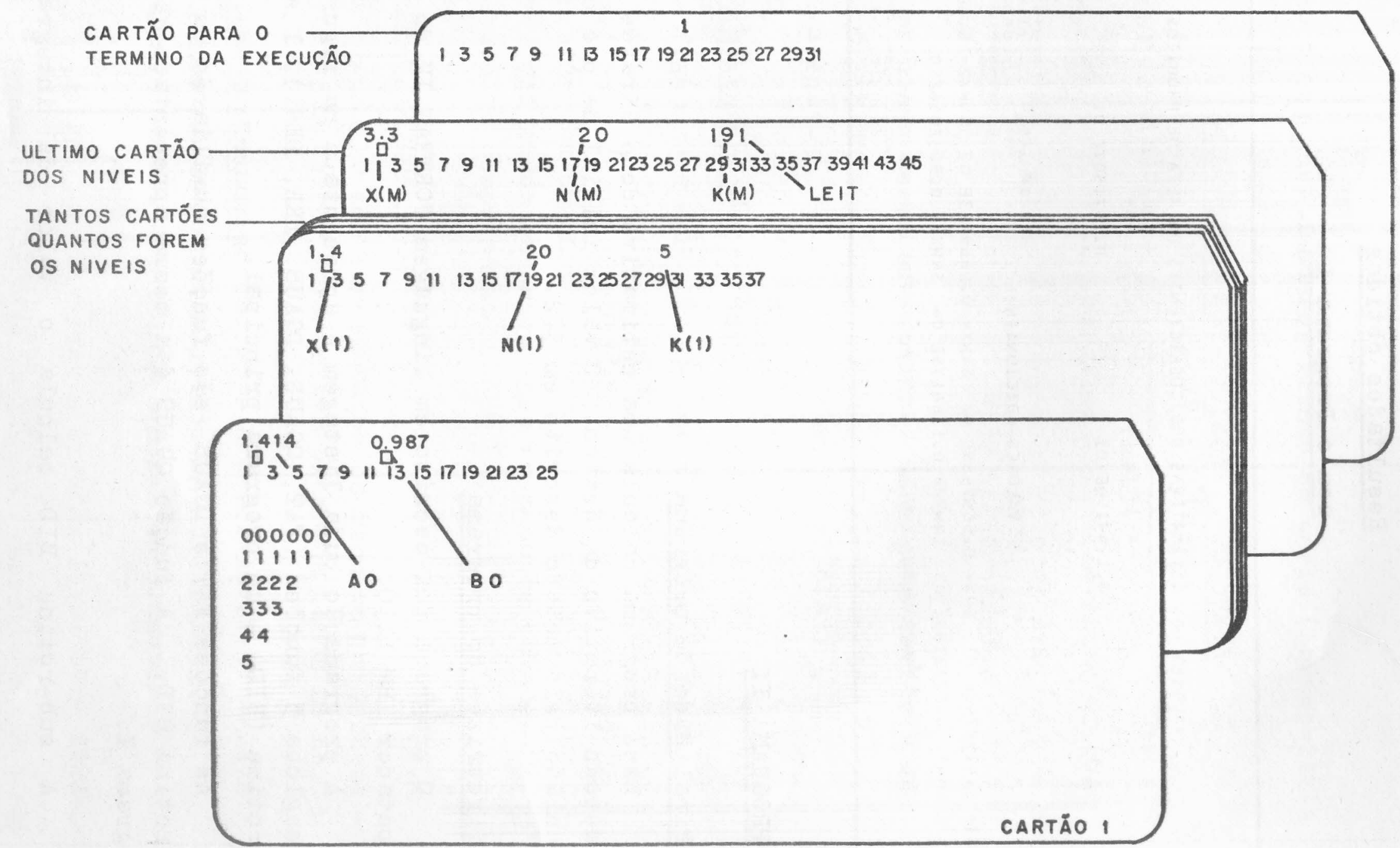




\section{Figura 6}

Resultados obtidos

\section{com o Programa I}

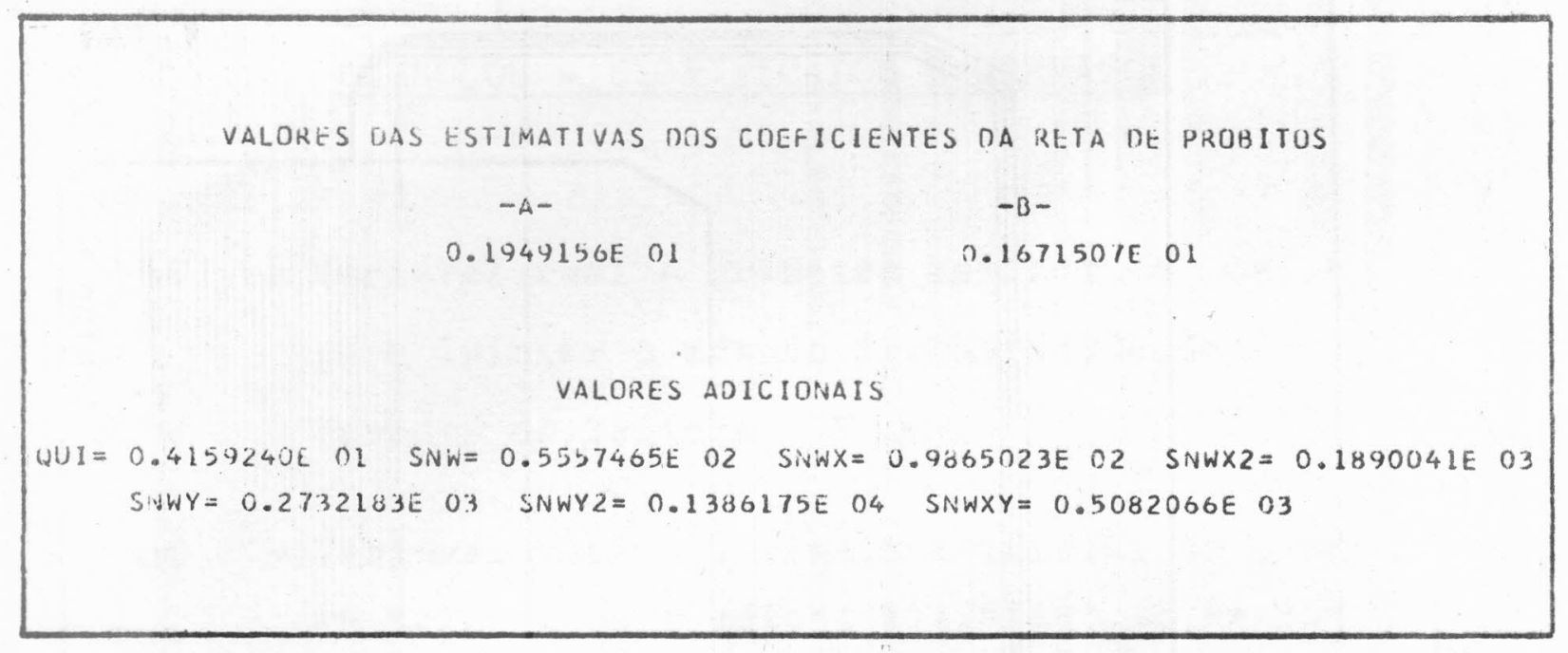

PROGRAMA II

Finalidade do programa

Este programa calcula uma estimativa de p através do método iterativo de Newton. O valor inicial de $\rho$ é calculado pelo método descrito em 3.2 .

\section{Diretrizes do programa}

o programa foi escrito em Iinguagem FORTRAN IV do com putador IBM 1130.

A programação cuja listagem é apresentacia na figura 7 engloba 5 funções (FAT, QGAUS, DGAUS, DER, DERI), 1 subrotina (BID) e um programa principal.

As funções FAT e DGAUS são funções auxiliares da sub rotina BID. A função QGAUS é a mesma apresentada no pro grama I.

A sub-rotina BID calcula o valor da integral 
$J(x, y, \rho)=\int_{x}^{+\infty} \int_{y}^{+\infty} \frac{1}{2 \pi \sqrt{1-\rho^{2}}} \exp \left[\frac{-1}{2\left(1-\rho^{2}\right)}\left(u^{2}+v^{2}-2 \rho u v\right)\right] d u d v$

usando o beguinte desenvolvimento em série (Abramowitz e Segun) [1]

$I(x, y, \rho)=Q(a) Q(x)+\sum_{n=0}^{\infty} \frac{z^{(n)}(x) \cdot z^{(n)}(y)}{(n+1) !} \rho^{n+1}$ em que $z(x)=\frac{1}{\sqrt{2 \pi}} e^{-\frac{x^{2}}{2}} \quad Q(x)=\int_{x}^{+\infty} z(t) d t$

A precisão dessa aub-rotina é dada pela varíavel TOL. As funcõer DER a DERI calculam $\partial I / \partial p$ e $\partial^{2} I / \partial \rho^{2}$, rezpectivamente.

\section{Dados necesgários}

Na figura 8 é apresentada uma tabela dos of́mbolos uti lizados para referenciar or dados, bem como as suar unddades. Na figura 9 é apresentado o formeto em que devem ser perfurados os cartões de dados, e a ordem em que devem aer colocados esaes cartões no maço de cartões que se rá levado a computador.

Resultados fornecidos

Na figura 10 aăo apresentadog os resultados forneci dos pelo computador para um dos exemplos dados no cap.3.

Precisão do Programa

A precisão do programa é fixada pelo uøuário. Na 11日tagem apresentada a precisão é lgual ou superior a um cen tésimo.

\section{Capacidade do Programa}

O programa apresentado na Iigura 7 permite o uso de até 100 nIveia Porém, eliterando-se o comando DIMENSION, a capacidade pode ser aumentada até o máximo permitido pela memória do computador. 


\section{Figure 7}

* LISTAGEM OO DECK * *

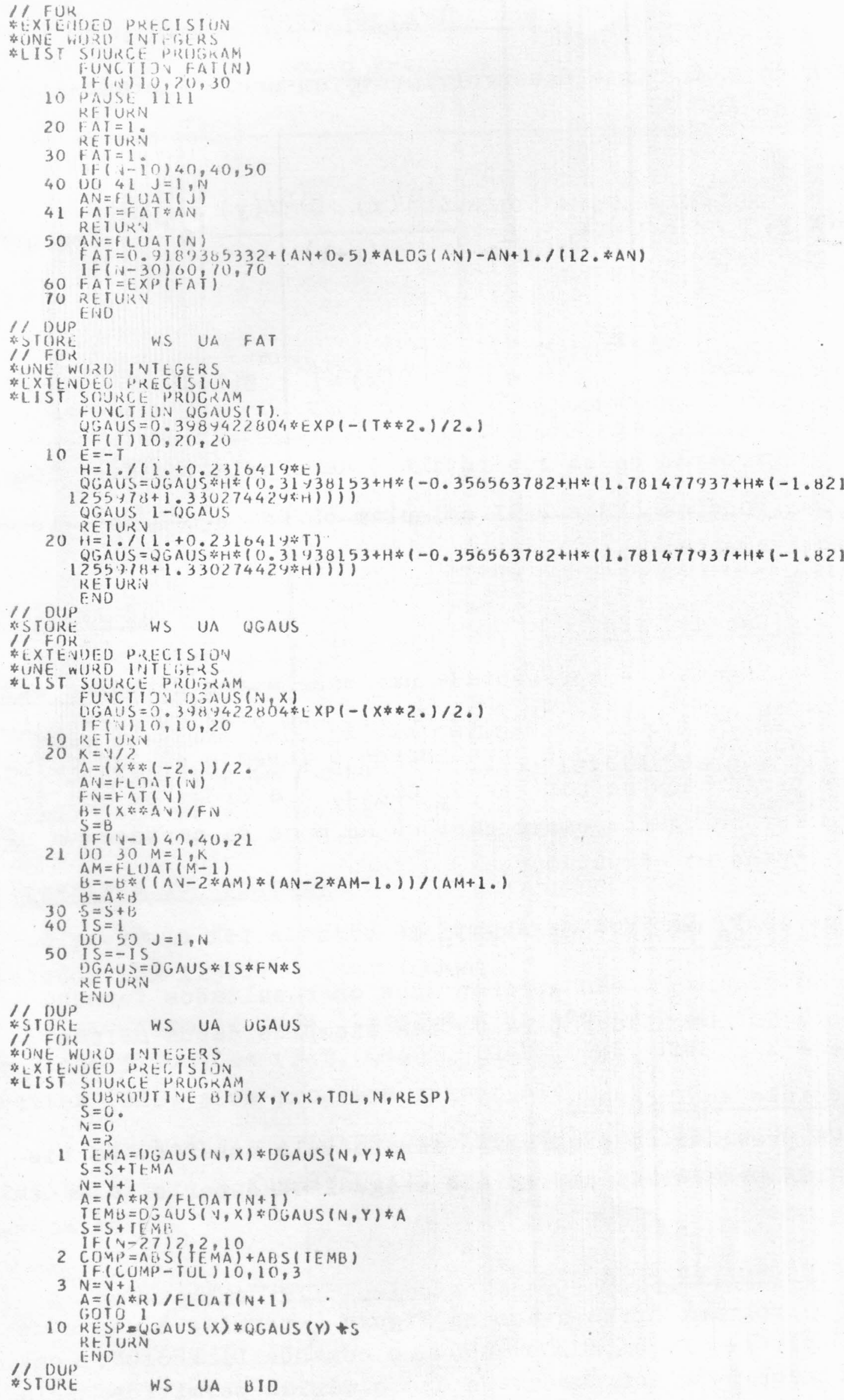


$11 \mathrm{FOK}$

* ETEVDFD PRECISIUN

*INE WGRD INTECERS

i.UNCTIOV DER(RO, $M, X, Y, N, K)$

DIMLNSILN X(I), YII), Ni I R K(1

TUL $=0.0 \cup 1$
() $E R=0$

(i) $I I=1, M$

$\left.P S_{I}=(0.1 \%) 1549431 / S_{0} R T(1-R O * 2)\right) * E X P((1-0.5) /(1-R O * 2)) *(X(1) *$

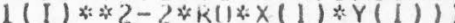

CALL BII) (Xi

$S=(N(1) * P S I \div(F L B A T(N)-K(I)) / N(I)-(1)) /(P * 0)$

1 DEK $D E R+S$.

$1 /$ DUP KI:TUiviv

* STUKL
$1 /$ FOK

WS UA DER

TEXTENDED PRECISIUIN

*UNE W(IRD) INTEGTRS

* List sOIURCE program

FUYCII J I T KI (KU, M, X, Y,N,K)

DIMLNSIIN X(1), Y(1), N(i), K(1)

$T D L=0.0 n)$

DERI $=0$

DO I I $=$

CALL BIUIX(I), Y(I), RO, TUL, NIT, O)

$P=1=(0.1591549431 / \operatorname{SON} T(1-R 0 * 2) * E X P(1) * 0.5) /(1-R 0 * 2)) *(X(1) * 2+Y$

(I) $* 2-2 * R(1) * x(1) * Y(I))$

$V=-(n(I) *(p)+2) /(p * 0)$

1 OERI $=$ DERI + V

*STDRE WS UA DERI

RETURN

* IOCS (CARD, 1132 PRINTER, TYPEWRITER, KEYBOARO,DISK, PLOTTER, 1403PRINTER)

* UNE WOSD TNIEGERS

* EXTE VIDE PRECISIUY

DIMENSIOV X(100),Y(100),N(100),K(100)

$T C L=0.001$

1 REAU( 2,$\}(10) \times M, Y M, D X, D Y, I F I M$

IF (IFIM) $9,10,9$

10 WRITE $(5,102) X_{4}, Y M, D X, D Y$ $M=0$

2. $M=M+1$

REAC, $(2,103) \times(M), Y(M), N(M), K(M)$

$X(M)=(X(: H)-X M) / P: X$

$Y(N)=(Y(N)-Y M) / D Y$

If $(v(M)) 3,3,2$

$3 \quad H=i-1$

DO 5 I 1,20

KO FLOAAT $(1-10) / 10$.

A ULR Kli,M, $x, Y, y, x$

COi $(5,4)$ ( 5 )

4 If (A) 6,6 ,

Continut:

CALL EXIT

$A=D$ t $R(K, M, X, Y, N, K)$

$B \geq D)=\{(K O, M, X, Y, N, K)$

$R B=R O-A / H$

IF $A B S(A / U-0.01) 1,1,6$

7 WRITE $(5,101) \mathrm{RO}$

$V=-1 . I$ I $R$ I ( $R O, M, X, Y, N, K)$

(105) 10

() $U$ I $=i$

Di) $8 \quad I=1, M$

CALL I?IU(X) (I),Y(I),Rก, TOL,IT, (U)

8 (JU) $=0 J U+(N(I) *(+\operatorname{LUA} T(N(I)-K(I)) / N(I)-Q) * 2) /(P * 0)$

$M=M-1$

WRITH $(5,104)$ QUI,

WhIT $(5,106)$

Gi) Tr. 1

1) CALL FXIT

100 FDRMAT $(4 F 10,0,15)$

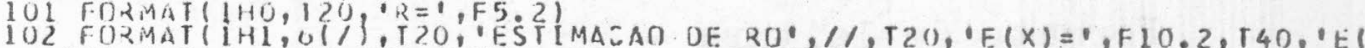

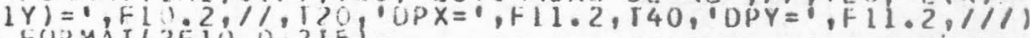

103 FURMATL 2 FIO.0.215

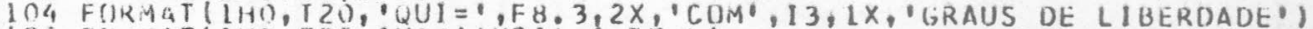

IOS FURMAT( IHO, T2O, V VARIANCIA $=$, F 7. S)

106 FORMATI

EIVD 


\section{Figura 8}

\section{SIMBOLOS - SIGNIFICADOS E FORMATOS}

XM - Variável real-média estimada do estímulo X Formato de leitura - F10.0

YM - Variável real - média estimada do estímulo Y

Formato de leitura. F 10.0

DX - Variável real - desvio padrão estimado do estímulo $X$

DY - Variável real - desvio padrão estimado do estímulo $Y$

I FIM - Variável inteira - variável que indica tór mino da execução do programa

Formato de leitura - I5

$X(M)$ - Variável real - nível do eatímulo X que en tra no m-ésimo nivel da mistura

Formato de leitura - F10.0

$Y(\mathbb{M})$ - Variável real - nivel do estimulo Y que en tra no m-ésimo nivel da mistura

Formato de leitura - F10.0

$N(M)$ - Variável real - tamanho do lote ao qual foi aplicado o m-ésimo nível da mistura. Formato de leitura - I 5

K(M) - Número de unidades experimentais que responderam ao m-ésimo nível da miatura. 
Fig. 9

DISPOSIÇÃO DOS DADOS DE ENTRADA DO PROGRAMA II

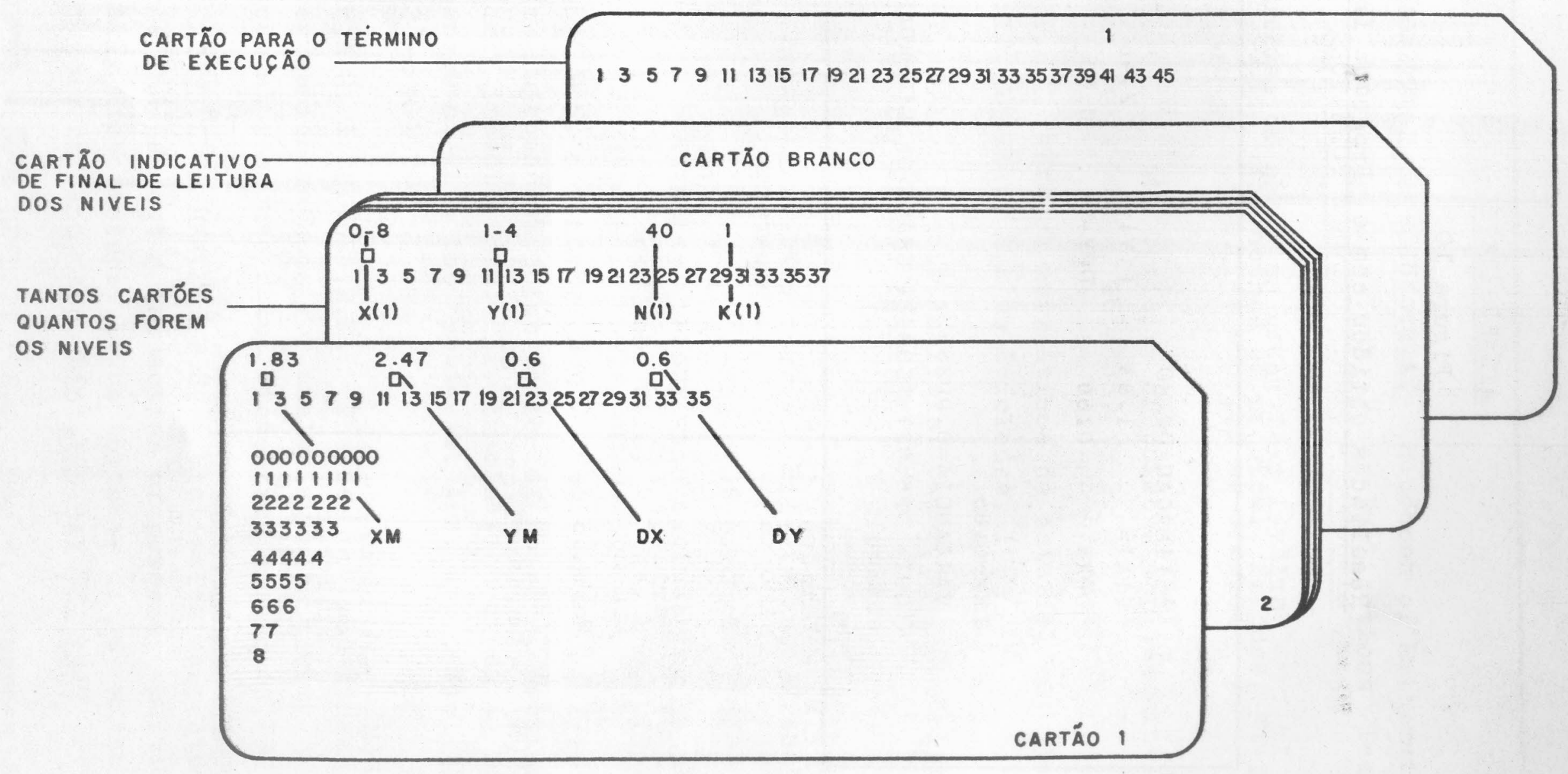


Figura 10

Resultados obtidos com o Programa II

$\begin{array}{lcll}\text { ESTIMACAO } & D E R O & & \\ E(X)= & 1.83 & E(Y)= & 2.46 \\ D P X= & 0.60 & D P Y= & 0.60\end{array}$

$R=-0.86$

VAR I ANC IA $=0.00266$

QUI $=25.027$ COM 31 GRAUS DE LIBERDADE 


\section{BIBLIOGRAFIA}

[1] ABRAMOWITZ, M. \& SEGUN, I.A. Handbook of Mathemati cal Functions. New York, Dover Publications Inc, p. $940,1969$.

[2] BLISS, C.I. The method of probits. Science, 79:38 $-9,1934$.

[3] BLISS, C.I. The toxicity of poisons applied fointly. Ann. Appl. Biol., 26, 585, 1939.

[4] CRAMER,H. Métoùos matemáticos de estatistica. Madrid, Aguilar, S.A de Ediciones, 1963

[5] FINNEY, D.J. Probit Analysis. Cambridge, Cambridge University Press, 1952.

[6] GARWOOD,F. The application of maximum likelihood to dosage-mortality curves. Biometrika, 32:46-58, 1941 .

[7] HALD,A. Statistical theory with engineering appli cations. New York, John Wiley \& Sons, Inc, 1952.

[8] HEWLETT,P.S. \& PLACKETT,R.D. Statistical aspects of the independent joint action of poisons, particularly insecticides. II. Examination of data for agreement with the hypotesis. Ann. Appl. Biol,37: $527-52,1950$.

[9] MOORE,R.H. \& ZEIGLER,R.K. The use of non-linear regression methods for analysing sensitivity and quantal response data. Biometrics,23:563-66,1967.

[10] PLACKETT,R.I. \& HEWLETT,P.S. Statistical aspects of the independent joint action of poisons, particu larly insecticides. I.The toxicity of a mixture of poisons. Ann. App. Biol, 35:347-58, 1948.

[1] PLACKETT, R.I. \& HEWLETT,P.S. Quantal responses to mixtures of poisons. J.R.Stat.Soc, 14(ㅌ):141-63, 1952. 
[12] WAID,A. Tests of statistical hypothesis conserning several parameters when the number of observations is large. Trans. Amer. Math. Soc, 54: $426-82,1943$. 